\title{
A New Interpretation of Vortex-Split Sudden Stratospheric Warmings in Terms of Equilibrium Statistical Mechanics ${ }^{\mathscr{O}}$
}

\author{
YUKI YASUDA ${ }^{\mathrm{a}}$ \\ Atmosphere and Ocean Research Institute, University of Tokyo, Kashiwa, Japan \\ FredDy Bouchet AND ANTOINE VenAille \\ Laboratoire de Physique, Université de Lyon, ENS de Lyon, Université de Claude Bernard, CNRS, Lyon, France
}

(Manuscript received 13 February 2017, in final form 20 August 2017)

\begin{abstract}
Vortex-split sudden stratospheric warmings (S-SSWs) are investigated by using the Japanese 55-year Reanalysis, a spherical barotropic quasigeostrophic (QG) model, and equilibrium statistical mechanics. The statistical mechanics theory predicts a large-scale steady state as the most probable outcome of turbulent stirring, and such a state can be computed without describing all the details of the dynamics. The theory is applied to a disk domain that is modeled on the polar cap north of $45^{\circ} \mathrm{N}$ in the stratosphere. The equilibrium state is obtained by computing the maximum of an entropy functional. In the range of parameters relevant to the winter stratosphere, this state is anticyclonic. By contrast, cyclonic states are quasi-stationary states corresponding to saddle points of the entropy functional. These results indicate that the mean state of the stratosphere associated with the polar vortex is not close to an equilibrium state but to a quasi-stationary state. The theoretical calculations are compared with the results of a quasi-static experiment in which a wavenumber-2 topographic amplitude is increased linearly and slowly with time. The results suggest that the S-SSW can be qualitatively interpreted as the transition from the cyclonic quasi-stationary state toward the anticyclonic equilibrium state. The polar vortex splits during the transition toward the equilibrium state.
\end{abstract}

\section{Introduction}

The Arctic polar vortex (hereafter the polar vortex) in the winter stratosphere is one of the largest coherent vortices in the atmosphere (e.g., Andrews et al. 1987; Haynes 2005; Waugh and Polvani 2010). The polar vortex is a cyclonic flow maintained through the thermal wind relation by radiative cooling and is perturbed by planetary-scale Rossby waves generated by topography and land-sea contrast in the troposphere. Sufficiently strong wave forcings sometimes break down the polar vortex. This extreme event has a time scale of a few days

\footnotetext{
${ }^{\text {a }}$ Current affiliation: Mitsubishi UFJ Morgan Stanley Securities Co., Ltd., Tokyo, Japan.

Supplemental information related to this paper is available at the Journals Online website: https://doi.org/10.1175/JAS-D-170045.s1.
}

Corresponding author: Yuki Yasuda, yuki.yasuda@17.alumni. u-tokyo.ac.jp and is accompanied by a reversal of zonal winds (from westerly to easterly) and a strong warming (sometimes over $50 \mathrm{~K}$ ) in the polar stratosphere, called a sudden stratospheric warming (SSW; e.g., Butler et al. 2015). The SSW is not only an interesting phenomenon but also practically important because it tends to affect tropospheric eddies (i.e., weather systems; e.g., Baldwin and Dunkerton 2001; Limpasuvan et al. 2004; Nakagawa and Yamazaki 2006; Charlton and Polvani 2007; Mitchell et al. 2013; Hitchcock and Simpson 2014; Kidston et al. 2015; Maycock and Hitchcock 2015). SSWs are classified into vortex-displacement type (D-SSWs) and vortexsplit type (S-SSWs) on the basis of the horizontal structure of the polar vortex (Charlton and Polvani 2007). In contrast with the D-SSW, during the S-SSW, the polar vortex has a nearly barotropic structure and splits into two daughter vortices almost simultaneously between the altitudes of about 20 and $40 \mathrm{~km}$ (Matthewman et al. 2009). Developing an understanding of the mechanism for SSWs remains an important theoretical subject. The aim of this study is to 
propose a new interpretation of S-SSWs with statistical mechanics arguments supported by numerical simulations.

Pioneering work on the mechanism for SSWs was made by Matsuno (1971). He proposed that SSWs result from the amplification of wave forcings to the mean flows. Following Matsuno (1971), SSWs have often been theoretically studied in the framework of wave-mean flow interactions. These studies may be roughly categorized into two groups. The first group employs a severely truncated dynamical system, called the HoltonMass model (Holton and Mass 1976). The second group focuses on the resonance of Rossby waves and is hereafter referred to as the resonance theory (e.g., Tung and Lindzen 1979a,b). Both types of studies rely on perturbation expansions in terms of a small parameter representing a normalized wave amplitude.

The Holton-Mass model describes interactions between a zonal mean flow, varying only in the vertical direction, and a single wave mode that also varies in the vertical direction and is generated by an effective bottom topography imitating tropospheric disturbances. As the topographic amplitude is increased, the model shows an abrupt transition from a quasi-steady state with a strong westerly wind (close to the radiative equilibrium) to a time-dependent state where the zonal wind vacillates between weak westerly and easterly (Holton and Mass 1976). Chao (1985) investigated the Holton-Mass model in terms of the catastrophe theory and argued that this transition (i.e., a catastrophe) corresponds to SSWs in the atmosphere. Similar abrupt transitions have been observed in a one-layer version of the HoltonMass model (Ruzmaikin et al. 2003; Birner and Williams 2008) and also in an improved Holton-Mass model in which the effective topography is specified through the Eliassen-Palm flux (Sjoberg and Birner 2014).

The second class of approaches (i.e., the resonance theories) may be further classified into linear and nonlinear theories. The linear theories have revealed that stationary Rossby waves can be resonant with topography in realistic velocity fields (Tung and Lindzen 1979a, b; Esler and Scott 2005). This means that an increase in the amplitude of an effective topography and also a resonance can lead to the wave amplification resulting in an SSW.

When a fluid system is near the resonant state, the wave amplitude is large, and nonlinear effects can no longer be ignored. Plumb (1981a,b) developed a weakly nonlinear theory based on a continuously stratified quasigeostrophic (QG) model and showed that the nonlinear effect makes a positive feedback; that is, the nonlinear adjustment to the mean velocity occurs in a way that amplifies the wave. Matthewman and Esler
(2011) developed Plumb's idea and investigated the onset of S-SSWs with a weakly nonlinear model. This model described the evolution of a barotropic vortex Rossby wave generated by a wavenumber-2 effective topography. They showed that an abrupt transition to a large-amplitude solution occurred in the model when the topographic amplitude was over the threshold, which corresponded to the evolution of a Rossby wave in the onset of an S-SSW. One should also see Esler and Matthewman (2011) for the application of these theoretical methods to D-SSWs.

An abrupt change in a large-scale flow structure, such as SSWs, is reminiscent of a phase transition in statistical mechanics. The equilibrium statistical mechanics for geophysical flows has made great progress over the last two decades (e.g., Salmon 1998; Majda and Wang 2006; Bouchet and Venaille 2012). Statistical mechanics is a powerful tool suited for the analysis of a nonlinear system with many degrees of freedom (e.g., QG system). One can build phase diagrams for macroscopic properties of a flow (e.g., the direction of the flow or the number of vortices) by computing the most probable state among all possible configurations without solving the governing equations. The most probable state is given as the solution of a variational problem and is referred to as the equilibrium state (EQS). By studying such phase diagrams and the properties of the equilibrium states, it is possible to understand the effects of varying external parameters, such as total energy and topographic amplitude. Statistical mechanics can be used for a strongly nonlinear system because it does not rely on the assumption of small-amplitude perturbations (or wave-mean flow decompositions). Conversely, statistical mechanics is applied only to a freely evolving flow without forcing or dissipation and does not indicate anything about the time evolution of the system, such as the relaxation toward the equilibrium state. However, it may be a natural first step to examine the following two questions:

1) Is the mean state of the stratosphere accompanied by the polar vortex close to an equilibrium state?

2) Is it possible to interpret S-SSWs (or D-SSWs) as a phase transition (i.e., a transition from an equilibrium state to another equilibrium state)?

This study tries to answer both questions.

The general theory of equilibrium statistical mechanics for geophysical flows is known as the MillerRobert-Sommeria (MRS) theory (Miller 1990; Robert 1991; Robert and Sommeria 1991). The MRS theory takes into account all conserved quantities, which give the constraints for the variational problem [i.e., the total energy and any moment of potential vorticity (PV)]. For 
simplicity, we employ a subclass of the MRS theory as a useful guide to interpret the results of numerical experiments. This subclass of the equilibrium theory gives a variational problem in which entropy is maximized under the constraints of two conserved quantities, the total energy and circulation (i.e., the first moment of PV; Chavanis and Sommeria 1996; Venaille and Bouchet 2009, 2011b; Naso et al. 2010). Any solution of this subtheory is a solution of the MRS theory, but the converse is not necessarily true (Bouchet 2008). This statistical mechanics theory will be introduced in section 3a.

Equilibrium statistical mechanics has already been applied to several geophysical fluid problems, including Jupiter's great red spots (Bouchet and Sommeria 2002), bottom-trapped flows over oceanic bottom topographies (Merryfield 1998; Venaille 2012), mesoscale eddies and jets in the ocean (Venaille and Bouchet 2011a), an idealized jet in a two-layer QG model (Esler 2008), and hurricanes (Prieto et al. 2001). Prieto and Schubert (2001) investigated the final states of the polar vortex evolving from dynamically unstable initial states by applying statistical mechanics to a spherical unforced barotropic model without effective topography.

The main results of this study are briefly summarized as follows. The mean state of the stratosphere associated with the cyclonic polar vortex is unlikely to be considered as an equilibrium state (i.e., the entropy maximum) but can be understood as the dominant quasi-stationary state, which is virtually a local maximum of the entropy. The term entropy refers to the number of possible (micro) states in a QG system. The S-SSW can be qualitatively interpreted as the transition from the (cyclonic) quasi-stationary state toward the (anticyclonic) equilibrium state.

To the best of our knowledge, there has been no study on SSWs explicitly using thermodynamic potentials, such as entropy and Helmholtz free energy. A phase transition is defined as a transition from an equilibrium state (i.e., a global maximum/minimum of some thermodynamic potential) to another equilibrium state or defined by a singularity of some thermodynamic potential. Although previous studies on SSWs may be implicitly influenced by equilibrium statistical mechanics, these studies do not rigorously show that SSWs can be interpreted as a phase transition (e.g., Chao 1985; Yoden 1987; Christiansen 2000; Monahan et al. 2003; Birner and Williams 2008; Matthewman and Esler 2011; Sjoberg and Birner 2014; Liu and Scott 2015).

This article is organized as follows. In section 2, we give a description of a barotropic QG model with a wavenumber- 2 effective topography. In section 3 , the statistical mechanics theory is applied to the barotropic
QG model on a disk domain. In section 4, to examine transitions among states of the polar vortex, a quasistatic experiment is performed with the spherical barotropic QG model, in which the amplitude of the wavenumber-2 effective topography is increased linearly and slowly with time. In section 5, using the theoretical calculations in section 3, we interpret the results of the quasi-static experiment and then propose a new interpretation of S-SSWs. Finally, the concluding remarks are given in section 6. D-SSWs are not investigated here because a stratified fluid model is necessary to describe them (Matthewman et al. 2009; Esler and Matthewman 2011). Independent of the statistical mechanics approach, we demonstrate in appendix A that the salient features of the observed S-SSWs can be captured in the framework of the barotropic QG model through direct comparison with a reanalysis dataset [Japanese 55-year Reanalysis (JRA-55)].

\section{Description of a barotropic QG model}

The S-SSW is known to have a nearly barotropic structure between the altitudes of about 20 and $40 \mathrm{~km}$ (Matthewman et al. 2009), and its dynamics has often been investigated by using one-layer fluid models with wavenumber-2 effective topographies (e.g., Matthewman and Esler 2011; Liu and Scott 2015; Scott 2016). Following these studies, we describe the dynamics over the altitude range of about $20-40 \mathrm{~km}$ by employing a barotropic QG model with a wavenumber-2 effective topography.

The governing equations (e.g., Vallis 2006) are

$$
\begin{aligned}
\partial_{t} q+\mathbf{v} \cdot \nabla q & =-\nu \Delta^{10} q \quad \text { and } \\
q(\mathbf{x}, t) \equiv & \Delta \psi(\mathbf{x}, t)+\underbrace{2 \Omega \sin \varphi}_{f(\mathbf{x})}+2 \Omega \sin \varphi h(\mathbf{x}, t) / H \\
& -\underbrace{2 \Omega \sin \varphi_{\text {off }}}_{f_{\text {off }}},
\end{aligned}
$$

where $q$ is the PV, $\psi$ is the streamfunction, $\mathbf{v}$ is the velocity given by $\mathbf{k} \times \nabla \psi$ ( $\mathbf{k}$ is a vertical unit vector), $\nu$ is a coefficient of hyperviscosity, $\Omega$ is the angular speed of Earth's rotation, $h$ is an effective bottom topography, and $H$ is an effective mean depth. Note that $h$ is considered to represent the effects of large-scale disturbances propagating from the troposphere, as in other theoretical models describing SSWs (e.g., Matsuno 1971; Holton and Mass 1976). The effective mean depth $H$ is set to the scale height of $6.14 \mathrm{~km}$ defined by $R_{\text {dry }} T_{0} / g$, where $R_{\text {dry }}$ is the gas constant for dry air $\left(287 \mathrm{~J} \mathrm{~K}^{-1} \mathrm{~kg}^{-1}\right), g$ is the gravity acceleration 



FIG. 1. Effective topographies over $0^{\circ}-90^{\circ} \mathrm{N}$ : (a) the zonal wavenumber-2 component of $h_{\mathrm{cmp}}\left(\equiv h_{\mathrm{cmp}, 2}\right)$ at the onset time made from JRA-55 and (b) $h$ with $a(t)=1$ defined in (3). The parameters $h_{0}, \lambda_{0}, \varphi_{0}$, and $\Delta \varphi$ in (3) are determined to fit $h$ into $h_{\mathrm{cmp}, 2}: h_{0}=420 \mathrm{~m}$, $\lambda_{0}=56.3^{\circ}, \varphi_{0}=58.1^{\circ} \mathrm{N}$, and $\Delta \varphi=13.0^{\circ}$. (c) Cross sections of (a) and (b) along the blue lines. In all panels, the magnitude of the effective topographies is normalized by $h_{0}(420 \mathrm{~m})$.

$\left(9.81 \mathrm{~m} \mathrm{~s}^{-2}\right)$, and $T_{0}(210 \mathrm{~K})$ is the climatological temperature for the midwinter over the polar cap north of $60^{\circ} \mathrm{N}$ in the JRA-55 [see Japan Meteorological Agency (2014), Kobayashi et al. (2015), and Harada et al. (2016) for details]. In the following, quantities except for time $t$ are basically nondimensionalized using the characteristic scales, such as 1 day, Earth radius, and mean depth. The above QG equations do not have a term of radiative relaxation because statistical mechanics does not incorporate external forcings. Effects of radiative cooling will be discussed in section 6 .

The theoretical calculations are performed on a disk domain (section 3), in which a position $\mathbf{x}$ is specified by a radius $r$ and an azimuthal angle $\theta$. In these calculations, $\nu$ is set to zero. By contrast, the numerical experiments are conducted on a sphere (section 4 ), where a position $\mathbf{x}$ is specified by a latitude $\phi$ and a longitude $\lambda$. The azimuthal angle $\theta$ on the disk is identical to the longitude $\lambda$ when the disk is obtained with Lambert's azimuthal equal-area projection: $r \equiv \sqrt{2(1-\sin \varphi)}$. The Coriolis parameter on the disk is obtained by projecting $2 \Omega \sin \varphi$. Lambert's projection is often used to identify S-SSWs in reanalysis datasets (e.g., Matthewman et al. 2009; Seviour et al. 2013).

The effective topography $h$ in (2) has the following form on the sphere:

$h \equiv a(t) \times h_{0} \cos \left[2\left(\lambda-\lambda_{0}\right)\right] \exp \left[-\frac{1}{2}\left(\frac{\varphi-\varphi_{0}}{\Delta \varphi}\right)^{2}\right]$.

For the theoretical calculations on the disk, $h$ is projected with Lambert's map and $a$ is regarded as a control parameter. To determine the parameters of $h$, we construct the composite potential temperature field associated with S-SSWs from JRA-55 with the method of
Seviour et al. (2013; see spp1.pdf in the supplemental material for details). The height field of the composite $550-\mathrm{K}$ surface, which is located at about $20 \mathrm{~km}$, is regarded as the effective bottom surface, and its zonal wavenumber- 2 component is denoted by $h_{\mathrm{cmp}, 2}$. The parameters of $h_{0}, \lambda_{0}, \varphi_{0}$, and $\Delta \varphi$ in (3) are determined to fit $h$ into $h_{\mathrm{cmp}, 2}$, as in the caption of Fig. 1, which compares $h(a=1)$ with $h_{\mathrm{cmp}, 2}$ (at the onset time). The simple form of $h$ represents well the structure of $h_{\mathrm{cmp}, 2}$ made from the composite analysis of JRA-55.

\section{Theoretical calculations based on equilibrium statistical mechanics}

In this section, a statistical mechanics theory is applied to the barotropic QG system on a disk domain. The main result is that the mean state of the stratosphere associated with the cyclonic polar vortex may be regarded as a quasistationary state (i.e., a saddle point of the entropy), while the equilibrium state (i.e., the maximum of the entropy) is an anticyclonic flow. This result suggests that the S-SSW may be qualitatively interpreted as a transition from the cyclonic quasi-stationary state. In section 5 , the theoretical calculations performed here will be compared with the results of numerical experiments.

\section{a. A variational problem given by equilibrium statistical mechanics}

An inviscid, freely evolving, two-dimensional flow tends to develop into finer and finer structures, while at a later time, it tends to reach a quasi-steady large-scale coherent structure. Equilibrium statistical mechanics gives a general method for obtaining a large-scale structure realized after strong PV mixing, without describing all the details of the dynamics (e.g., Salmon 1998; Majda and Wang 2006; 
Bouchet and Venaille 2012). Statistical mechanics is a static theory, as it does not give any information on the dynamics, such as the time evolution during a transition. It should also be pointed out that the statistical mechanics applies only to an isolated fluid system without dissipation or forcing. In forced-dissipative cases, the theory may apply only if the inertial time scale (for instance, given by the eddy turnover time) is much smaller than the dissipation and forcing time scales.

The present study employs the following variational problem (Chavanis and Sommeria 1996; Venaille and Bouchet 2009, 2011b; Naso et al. 2010), which belongs to a subclass of the more general Miller-RobertSommeria theory (section 1$)$ :

$$
\max _{q}\{S \mid E, \Gamma\}
$$

where

$$
\begin{aligned}
\Gamma & \equiv \int q d A, \\
E & \equiv \frac{1}{2} \int(\nabla \psi)^{2} d A, \text { and } \\
S & \equiv-\frac{1}{2} \int q^{2} d A .
\end{aligned}
$$

Here, $d A$ is the area element, $\Gamma$ is the total PV, $E$ is the total energy, and $S$ is the negative of potential enstrophy. The surface integrals in (5)-(7) are taken over the whole domain. Equation (4) means that $S$ is maximized by varying $q$ subject to the two constraints of constant $E$ and $\Gamma$. The solution $q$ of (4) is referred to as the equilibrium state. One of the most important points is that an overwhelming number of possible configurations are associated with the equilibrium state. This means that if one state were picked up at random among all possible states and if a spatial coarse graining were performed, the equilibrium state would be recovered. Hereafter, the negative of potential enstrophy $S$ is called entropy. The entropy $S$ has a one-to-one correspondence to the mixing entropy, when a probability density function of PV is Gaussian, as in our case considered here (Naso et al. 2010). A brief explanation of this result is given in appendix Ba.

Any stationary point, that is, a state with the first-order variation of $S$ being zero under the two constraints of $E$ and $\Gamma$, satisfies the following linear $q-\psi$ relationship:

$$
q=b \psi-c
$$

where $b$ and $c$ are Lagrange multipliers, depending implicitly on the two constraints of $E$ and $\Gamma$. A derivation of
(8) is given in appendix $\mathrm{Bb}$. This relationship means that a streamline is identical to the corresponding PV contour; therefore, any stationary point for (4) is an exact steady solution of the QG system [(1) and (2), where $\nu=0$ ]. In addition to the entropy maximum (i.e., the equilibrium state), we focus on a local maximum and a saddle point of $S$, which are referred to as a metastable and a quasi-stationary state, respectively. The equilibrium and metastable states are dynamically and nonlinearly stable against any small-amplitude perturbation, but the quasi-stationary states are not necessarily stable and may be destabilized by some perturbation (Ellis et al. 2002; Naso et al. 2010; Venaille and Bouchet 2011b). This is the reason for referring to the latter as "quasi"-stationary states.

\section{b. Equilibrium and quasi-stationary states}

The aim of this subsection is to show that the equilibrium state is anticyclonic, whereas quasi-stationary states are cyclonic, with a realistic parameter set for the winter stratosphere.

We first need to determine a domain over which the variational problem (4) is applied. For the moment, we focus on the polar cap over $45^{\circ}-90^{\circ} \mathrm{N}$ because significant changes in a flow field are often observed over the North Pole during an S-SSW (e.g., Matthewman et al. 2009). In section $5 \mathrm{c}$, on the basis of a numerical QG solution, an appropriate polar cap is determined to make quantitative comparisons between the theory and the numerical experiment.

Assuming this polar cap to be a closed domain, we propose now to compute the equilibrium and quasistationary states within this domain by solving the variational problem (4). These states are easily expressed in terms of Laplacian eigenmodes (Chavanis and Sommeria 1996; Venaille and Bouchet 2009, 2011b; Naso et al. 2010). However, computing the Laplacian eigenmodes over a polar cap (i.e., a part of the sphere) leads to unnecessary technical difficulties because the eigenmodes do not have a simple analytic expression, such as spherical harmonics. For this reason, we consider a simpler geometry, namely, a disk, which is obtained by projecting the polar cap north of $45^{\circ} \mathrm{N}$ onto the plane with Lambert's azimuthal equal-area projection: $r=\sqrt{2(1-\sin \varphi)}$ and $\theta=\lambda$. The values of $\Gamma, E$, and $S$ are invariant under Lambert's projection because they are the surface integrals of scalars, and Lambert's projection preserves the area element. This point facilitates the comparisons of the theoretical calculations with the simulation results (section 5).

The Coriolis parameter $f$ and the effective topography $h$ are also projected with Lambert's map. The projected $f$ is a monotonic function having a maximum at the origin 

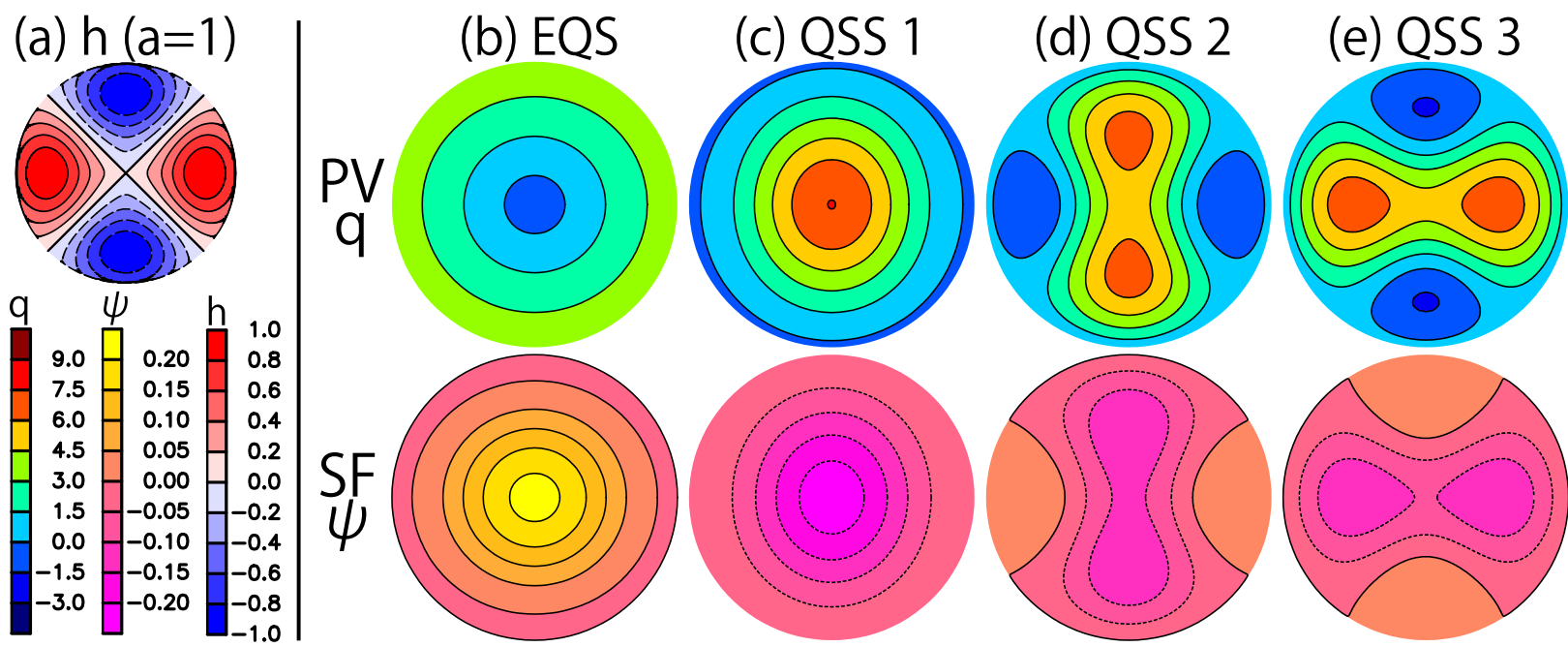

FIG. 2. (a) Effective topography $h$ over $45^{\circ}-90^{\circ} \mathrm{N}$ projected onto the plane with Lambert's azimuthal equal-area projection, where $\lambda_{0}$ in (3) is set to zero. (b)-(e) Theoretically calculated PV $q$ and streamfunction (SF) $\psi$ of (b) equilibrium state (EQS), (c) QSS 1, (d) QSS 2, and (e) QSS 3, where QSS $n$ stands for the quasi-stationary state having the $n$th largest $b$ in (8). The parameters are fixed to $(a, \Gamma, E)=(0.15,4.2,0.10)$.

$(r=0)$, which corresponds to the North Pole. The effect of Earth's curvature is partially taken into account, even though we consider a disk on a plane. The spatial structure of $h$ is fixed, while its amplitude is controlled by $a$ [see (3)]. The projected $h$ with $a=1$ is shown in Fig. 2a, where $\lambda_{0}$ in (3) is set to zero, without loss of generality.

A stationary point for the variational problem (4) is determined by the three parameters, ${ }^{1}$ the topographic amplitude $a$, the total PV $\Gamma$, and the total energy $E$. The calculation method to obtain the equilibrium and quasistationary states is described in appendix $\mathrm{C}$, and the expressions of the Laplacian eigenmodes are given in appendix D.

Equilibrium and quasi-stationary states are obtained for all possible values of the parameters (i.e., $a, \Gamma$, and $E$ ) without solving the QG equations [(1) and (2)]. However, since some of these values are irrelevant to the winter stratosphere, the parameters have to be confined to an appropriate range. In this subsection, the parameters are fixed to $(a, \Gamma, E)=(0.15,4.2,0.10)$, which are close to the values when the cyclonic polar vortex exists in the numerical experiments (section 4). These experiments are based on the climatological fields made from JRA-55, which indicates that the above parameter

\footnotetext{
${ }^{1}$ Because time does not matter for the variational problem (4), it is always possible to choose a time unit such that $E=1$. Therefore, we are left with only two parameters, the topographic amplitude $a$ and the total PV $\Gamma$. However, the transformation of the time unit makes it a little difficult to compare the theoretical calculations with the simulation results. Therefore, we consider the three parameters of $a, \Gamma$, and $E$
}

values are in a range relevant to the winter stratosphere. In the next subsection, the three parameters are varied.

As in the above discussions, the size of a domain and the values of parameters (e.g., the total energy) need to be obtained from a QG solution (or a reanalysis dataset), when a specific phenomenon is examined by using statistical mechanics. See Chavanis and Sommeria (1996), Venaille and Bouchet (2009, 2011b), and Naso et al. (2010) for general discussions on the QG systems in terms of equilibrium states and phase diagrams without solving the QG equations; in particular, Chavanis and Sommeria (1996) investigated a phase diagram for a barotropic fluid on a disk domain.

Figure $2 \mathrm{~b}$ shows the equilibrium PV and streamfunction. The PV is minimum at the origin (corresponding to the North Pole), but the streamfunction is maximum there, which means that the equilibrium state is anticyclonic. Therefore, the mean state of the stratosphere accompanied by the cyclonic polar vortex may not be considered as an equilibrium state. In other words, even when $\Gamma$ and $E$ are high enough to form a strong (cyclonic) polar vortex, the equilibrium state (i.e., the entropy maximum) is anticyclonic.

Venaille and Bouchet (2011b) and Naso et al. (2010) pointed out that a cyclonic state may exist as a quasistationary (or metastable) state, even when the equilibrium state is anticyclonic. For the variational problem (4), there are infinitely many quasi-stationary states, where small-scale structures become dominant, as the inclination $b$ in (8) is decreased (Chavanis and Sommeria 1996). We investigate here a few quasistationary states having large $b$. Figures $2 \mathrm{c}-\mathrm{e}$ show the 


\section{Domain boundaries of existence}

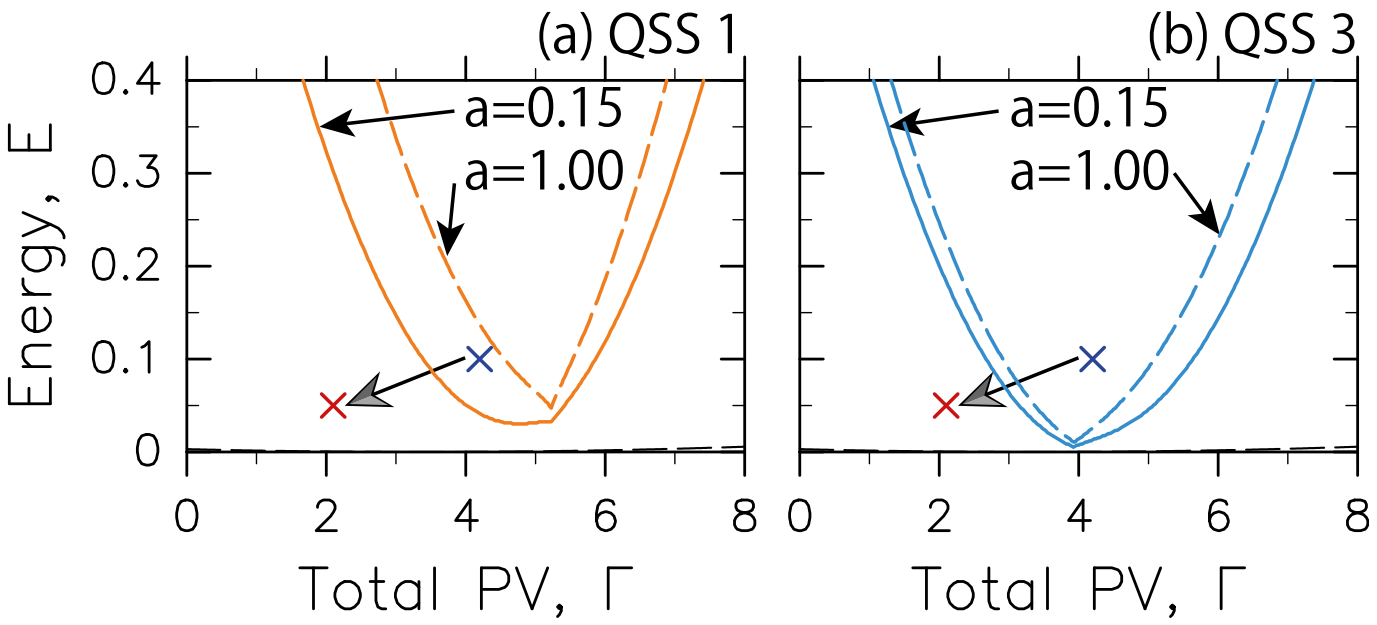

FIG. 3. Theoretically calculated domain boundaries of existence of (a) QSS 1 and (b) QSS 3 in the $\Gamma-E$ space with the topographic amplitude $a=0.15$ (solid) and $a=1.00$ (dashed). The domain boundary for the equilibrium state is also shown by the dashed black curve, where $a=1.00$. Each state exists at higher energies than those on the boundary. In each panel, the blue cross is at $(\Gamma, E)=(4.2,0.10)$ and the red cross is at $(\Gamma, E)=(2.1,0.05)$.

three quasi-stationary states (QSS), where QSS $n$ stands for the quasi-stationary state having the $n$th largest $b$. The three quasi-stationary states are cyclonic, as seen in their streamfunctions. The PV patch of QSS 3 is laterally long, but the PV patch of QSS 1 or 2 is elongated along the direction perpendicular to that of QSS 3. QSS 1 has the largest structure because of the largest $b$, and its PV is a maximum at the origin (corresponding to the North Pole). Therefore, QSS 1 appears to be similar to the mean state of the winter stratosphere, where the polar region is covered with the cyclonic polar vortex.

QSS 1 is a special saddle point of the entropy $S$, but QSS 2 and 3 are saddle points without such a property. Expressing the entropy in terms of a quadratic form, we can investigate the structure of the entropy surface in phase space (see appendix E for details). Roughly speaking, QSS 1 is virtually a local maximum of $S$, and the dynamical stability against almost all smallamplitude perturbations is theoretically assured. More precisely, in phase space, the entropy of QSS 1 is increased only along the two directions of the largest Laplacian eigenmodes with azimuthal wavenumber 1. In other words, QSS 1 may be destabilized only when a perturbation has these wavenumber- 1 components. Note that there are two different eigenmodes because of the degeneracy in a disk domain (appendix D). Moreover, in a general domain without symmetry, such as a rectangular domain, QSS 1 can be a local maximum of the entropy (i.e., metastable; Naso et al. 2010). In this case, the dynamical stability of QSS 1 against any smallamplitude perturbation is theoretically assured.

\section{c. Domains of existence of quasi-stationary states}

The results in the previous subsection are at one parameter point $[(a, \Gamma, E)=(0.15,4.2,0.10)]$. Similar results are obtained over the parameter range of the numerical experiments in section 4; however, some quasi-stationary states do not exist in some parameter domains. In this subsection, we examine the parameter domains for the existence of QSS 1,2, and 3.

Figure 3 shows the domain boundaries of existence of QSS 1 and 3 in the $\Gamma-E$ space. Each state exists at higher energies than those on the colored curves (solid for $a=0.15$ and dashed for $a=1.00$ ). The method of obtaining these boundaries is described in appendix C. The domain of existence of QSS 2 is the same as that of QSS 1, because both states annihilate at the same parameters. The domains of existence of QSS 1 and 3 become narrower and shift to regions with higher energies as the topographic amplitude $a$ is increased. The domain boundary for the equilibrium state is also shown by the dashed black curve, which nearly overlaps with the $\Gamma$ axis in each panel. This black curve is almost independent of $a$, and the curve with $a=1.00$ is shown.

In Fig. 3, the blue cross is at $(\Gamma, E)=(4.2,0.10)$, which is close to the values when the cyclonic polar vortex exists over the North Pole (section 4). After an S-SSW, the polar vortex will become quite weak, which suggests that the values of $\Gamma$ and $E$ significantly decrease. For instance, we assume that both values vary to $(\Gamma, E)=(2.1,0.05)$, denoted by the red cross in Fig. 3. During this variation, the domain boundaries of 
existence of QSS 1 and 3 are crossed. In this case, if the initial state is QSS 1 (or 3), a transition will occur because the parameters enter the domain without both states. This suggests that the S-SSW may be interpreted as a transition from QSS 1 (or 3). It is worth noting that equilibrium statistical mechanics does not give any information on time evolution, such as the relaxation toward the equilibrium state. This also means that statistical mechanics does not predict the variations of the total PV $\Gamma$ or energy $E$, while these variations are obtained by solving the $\mathrm{QG}$ equations and then externally used in the statistical mechanics theory. A careful analysis of simulation results is necessary to discuss a transition between two states. We perform this analysis in section 5 .

\section{Numerical experiments with a spherical QG model}

In the previous section, we suggest that the S-SSW may be interpreted as a transition from a cyclonic quasistationary state. However, the final state after the transition is unknown because equilibrium statistical mechanics does not indicate anything about time evolution. In this section, we perform numerical experiments to reveal transitions associated with S-SSWs. These results will be interpreted with statistical mechanics in the next section.

When we conduct a numerical experiment based on the real S-SSWs, the details of transitions (or bifurcations) of the polar vortex are not clear because the real S-SSWs have a time scale of a few days and the time evolution is too rapid (see appendix A). It may be useful for understanding S-SSWs to carry out an experiment with a slowly varying topography and to pursue changes in the structure of a flow field. In fact, several studies (e.g., Matthewman and Esler 2011; Liu and Scott 2015) have argued that some transition is essential to interpret S-SSWs. To examine such a transition, we perform a quasi-static experiment where the time scale of the effective topography $h$ is $O\left(10^{4}\right)$ days. Because of this quite slow variation of $h$, the flow field is nearly steady, and it is possible to reveal the states before and after a transition.

A link of the real S-SSWs to the quasi-static experiment, in which the time scale of $h$ is unrealistically long, may be unclear. Before conducting such a highly idealized simulation, we perform an experiment with the time scale of $h$ being 1 week, which is a reasonable time scale for Rossby waves causing S-SSWs (Sjoberg and Birner 2012).

We first describe the model configuration common to the experiments with short and rather long time scales.
The effective topography $h$ is given by (3), where its amplitude $a(t)$ is varied with time:

$$
a(t) \equiv\left\{\begin{array}{cl}
0 & (t \leq 0), \\
a_{\max } \frac{t}{\Delta t} & (0<t \leq \Delta t), \\
a_{\max } & (\Delta t<t) .
\end{array}\right.
$$

The parameters $a_{\max }$ and $\Delta t$ will be given in the following subsections.

In the atmosphere, the mean state of the winter stratosphere results from the balance between radiative cooling and eddy diffusion (e.g., Haynes 2005; Waugh and Polvani 2010). The QG model (1) and (2) cannot form such a balanced state because it does not incorporate radiative relaxation. Thus, the initial balanced state is made from JRA-55 and given to the QG model. More precisely, the initial PV is the axisymmetric component of the climatological barotropic absolute vorticity $\Delta \psi+f$, which is determined by the following two steps. In step 1 the climatological (three dimensional) absolute vorticity is obtained by simply averaging the absolute vorticity over the 56 midwinters (December-February from $1958 / 59$ to 2013/14) in JRA-55, except for the periods of SSWs. In step 2 its barotropic component is defined by vertically averaging the obtained absolute vorticity with a weight of density over the potential temperature surfaces of 550-1300 K (about $22-39 \mathrm{~km}$ ). Note that SSWs (i.e., D-SSWs and S-SSWs) are identified with the method of Seviour et al. (2013; see spp1.pdf in the supplemental material for details).

The spherical QG equations [(1) and (2)] are solved with the fourth-order Runge-Kutta method and the spectral method with the spherical harmonic expansion. $^{2}$ All experiments were conducted with the truncation wavenumber (in a triangular manner) T106 with a time step of $12 \mathrm{~min}$. When calculating the nonlinear terms, we use the standard transform method with an alias-free grid of 320 (zonal) $\times 160$ (meridional). Some experiments were also performed with $\mathrm{T} 63, \mathrm{~T} 85$, and $\mathrm{T} 126$ to confirm the insensitivity to the truncation wavenumber. The viscosity coefficient $\nu$ was chosen such that the $e$-folding time for the modes with the highest total wavenumber was either 80,8 , or $2.4 \mathrm{~h}$. We confirmed that all results are not highly sensitive to $\nu$.

\footnotetext{
${ }^{2}$ The numerical model was constructed with a library (ISPACK) made by Ishioka (2013).
} 
(a) Time series of amp. $h$



(b) Snapshots of PV $\left(45-90^{\circ} \mathrm{N}\right)$

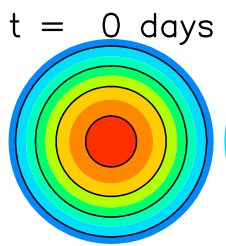

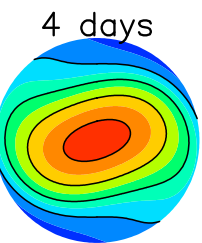

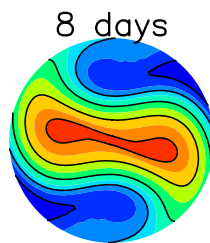

12 days

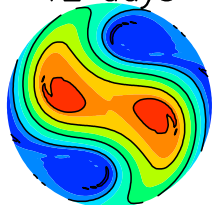

FIG. 4. (a) Time series of the topographic amplitudes given by $h_{\mathrm{cmp}, 2}$ (red) made from JRA-55 and by $h$ (black) in (3) and (9), where $\Delta t=$ 7 days and $a_{\max }=2$. The same effective topography $h_{\mathrm{cmp}, 2}$ as that in Fig. A1d is used for the red time series. For comparison, the onset time is shifted to $t=10$ days. (b) Evolution of PV over $45^{\circ}-90^{\circ} \mathrm{N}$ simulated by the QG model using $h$ in (a).

\section{a. Experiment with time scale of 1 week}

In this subsection, we conduct an experiment with the time scale $\Delta t=7$ days, where $a_{\max }$ in (9) is set to 2 . Figure 4a compares the time series of $a(t)$ in (9) with that of the amplitude of $h_{\mathrm{cmp}, 2}$, which is made through the composite analysis of JRA-55 (section 2 and appendix A). The time scale and amplitude of $h$ are similar to those of $h_{\text {cmp }, 2}$.

Figure $4 \mathrm{~b}$ shows the simulated PV evolution. The vortex splitting is reproduced even by using the simple effective topography $h$ in (3) and (9). This PV evolution is similar to those observed in more realistic experiments (see Figs. A1c,e). These results motivate us to further investigate the transitions in the QG model by performing an experiment with a large $\Delta t$ (i.e., the amplitude of $h$ is slowly increased).

\section{b. Quasi-static experiment with time scale of $\mathrm{O}\left(10^{4}\right)$ days}

We conduct a quasi-static experiment, where $\Delta t$ and $a_{\max }$ in (9) are set to $2.8 \times 10^{4}$ days and 1 , respectively. The following results are insensitive to $\Delta t$. Similar results were obtained when $\Delta t=1.4 \times 10^{4}$ days, which indicates that the variation in the topographic amplitude $a(t)$ is sufficiently slow. Without loss of generality, $\lambda_{0}$ in (3) is set to zero (see Figs. 1b and 5c). We examine the evolution of the PV field and the variations of the integral quantities, $\Gamma, E$, and $S$, defined by (5), (6), and (7), respectively. The surface integrals in (5)-(7) are taken over the polar cap north of $45^{\circ} \mathrm{N}$.

The PV field is nearly steady except around the two transitions. ${ }^{3}$ Figure 5 shows the time series of $\Gamma, E, S$, and the major-axis angle of the equivalent ellipse (Fig. 5a); the typical snapshots of the three nearly steady states (Fig. 5b); and the structure of the

\footnotetext{
${ }^{3}$ See spp2.gif in the supplemental material for the evolution of $\mathrm{PV}$ and streamfunction from the start to the end of the quasi-static experiment.
}

effective topography $h$ (Fig. 5c). The equivalent ellipse is obtained by following Matthewman and Esler (2011), which represents well the edge of the polar vortex. The changes in the PV field are correlated with the abrupt changes in $\Gamma, E$, and $S$. The first transition occurs at about $t=7000$ days. The state before the transition is named $A$, in which the polar vortex is vertically elongated (i.e., the major-axis angle $\sim 0^{\circ}$ ), as shown in the snapshots at day 4000 . The state after the transition is named $\mathrm{B}$, in which the polar vortex is laterally elongated (i.e., the major-axis angle $\sim 90^{\circ}$ ), as shown in the snapshots at day 11000 . Associated with the transition from A to B, the values of $\Gamma, E$, and $S$ rapidly decrease. Note that the temporary increase in the major-axis angle around $t=5000$ days (Fig. 5a) was not observed in some experiments using a different viscosity coefficient or truncation wavenumber, and it is not discussed here.

The major-axis angle of the equivalent ellipse increases before about $t=2000$ days and then reduces to about $0^{\circ}$ (Fig. 5a). This variation is sensitive to a change in the initial PV (not shown). This is likely because the aspect ratio of the equivalent ellipse is close to unity (i.e., the ellipse is almost a circle) and there is a high uncertainty in the direction of its major axis, which will lead to the sensitivity of the major-axis angle. During this period, the initial axisymmetric state changes into A, which is not axisymmetric (Fig. 5b). For simplicity, we regard the initial axisymmetric state as $\mathrm{A}$. When the topographic time scale $\Delta t$ in (9) is 7 days and the initial PV is given by that of state A (i.e., the PV at day 4000 in Fig. 5b), a vortex splitting similar to that in Fig. $4 \mathrm{~b}$ is reproduced (not shown). Furthermore, when $\Delta t$ is $2.8 \times 10^{4}$ days (quasi static) and the initial PV includes all zonal wavenumber components (not axisymmetric), a state similar to A appears and persists for about 3000 days (not shown). These results imply that the emergence and persistence of state $\mathrm{A}$ is not highly sensitive to the initial PV and validate that the initial state is regarded as $\mathrm{A}$. 


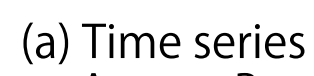

(b) SF $\psi$ and PV q $\left(45-90^{\circ} \mathrm{N}\right)$

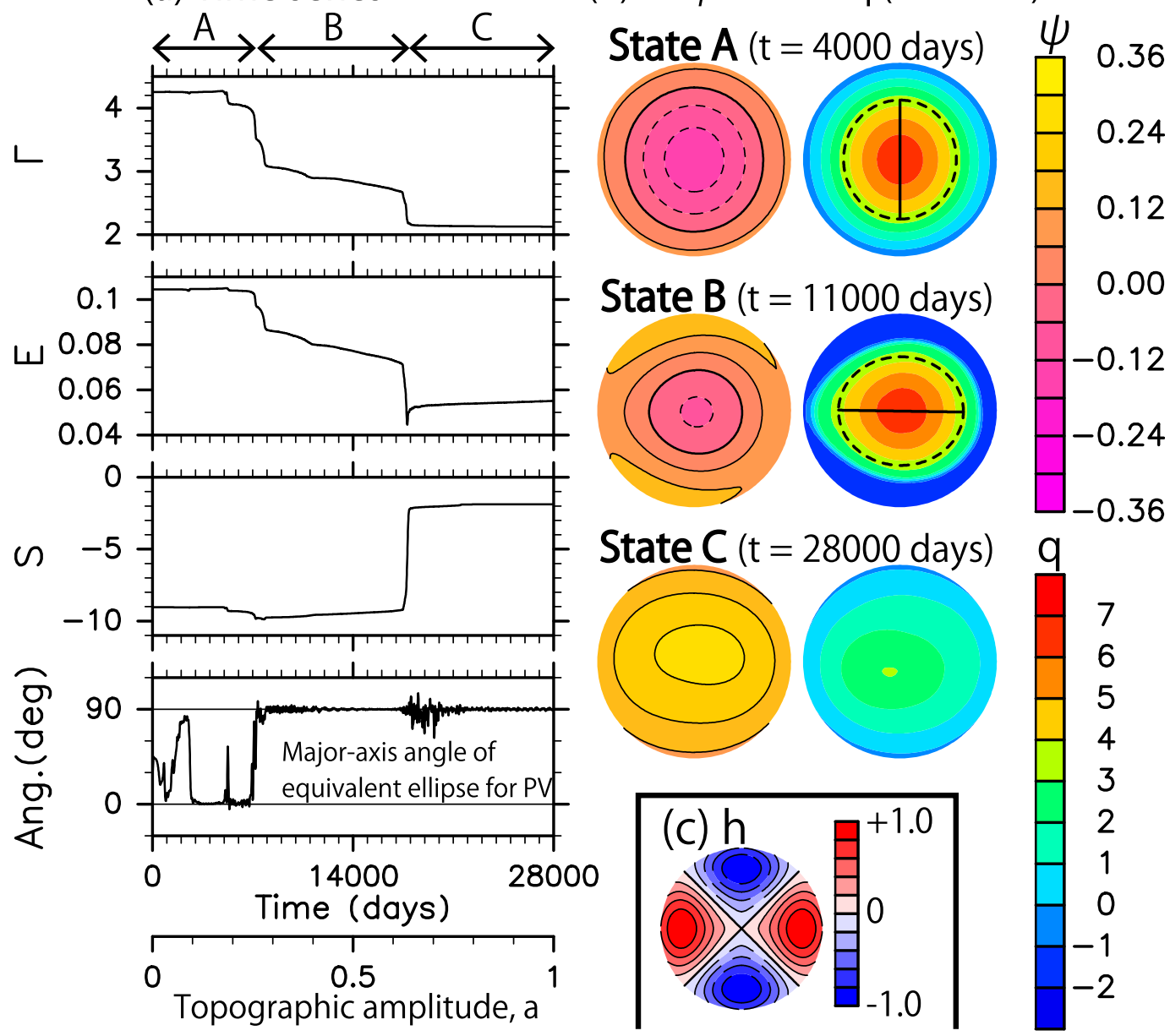

FIG. 5. Results of the quasi-static experiment with the QG model using $h$ in (3) and (9), where $\Delta t=2.8 \times 10^{4}$ days and $a_{\max }=1$. (a) Time series of the total PV $\Gamma$, the total energy $E$, the entropy $S$, and the major-axis angle of the equivalent ellipse. The quantities of $\Gamma, E$, and $S$ are defined in (5), (6), and (7), respectively. The equivalent ellipse is obtained by following Matthewman and Esler (2011), where its major-axis angle is measured from the vertical axis in the clockwise direction. A low-pass filter with a 100-day cutoff period was applied to all time series (the results are insensitive to the cutoff period). (b) Typical snapshots over $45^{\circ}-90^{\circ} \mathrm{N}$ of the three nearly steady states observed in the quasi-static experiment. SF means streamfunction $\psi$. In the top and middle PV snapshots, the equivalent ellipses are drawn by the dashed curves and their major axes by the solid lines. In (a), the duration of each nearly steady state is roughly denoted by the arrow. See text for details. (c) Effective topography $h$ with $a(t)=1$ over $45^{\circ}-90^{\circ} \mathrm{N}$. The parameters of $h$ are the same as those in Fig. 1b, except for $\lambda_{0}=0^{\circ}$.

The second transition occurs at about $t=18000$ days, accompanied by the polar vortex collapse. The PV filaments are peeled off the polar vortex, and eventually the polar vortex breaks down without splitting. ${ }^{4}$ Before this transition, the flow field is at state $\mathrm{B}$. The state after the transition is named $\mathrm{C}$, in which the weak PV patch is laterally elongated, as shown in the snapshots at day 28000 (Fig. 5b). Associated with the transition from B to

\footnotetext{
${ }^{4}$ See spp3.gif in the supplemental material for the evolution of $\mathrm{PV}$ and streamfunction around the vortex collapse.
}

C, the values of $\Gamma$ and $E$ rapidly decrease but that of $S$ rapidly increases because of the strong PV mixing (Fig. 5a). Just after the transition, the small PV patch remains, but it is dissipated at about $t=21500$ days.

More importantly, state B (as well as A) is cyclonic, whereas $\mathrm{C}$ is anticyclonic, as seen in the streamfunctions (Fig. 5b). Note that $\mathrm{C}$ is anticyclonic, independent of the small PV patch left after the vortex breakdown. A similar flow field to that of state $\mathrm{C}$ was also obtained in the experiment in the previous subsection, where $\Delta t=7$ days, sufficiently after the vortex splitting (not shown). These results indicate that the final state $\mathrm{C}$ is not 
sensitive to the topographic time scale $\Delta t$, but the vortex splitting and the emergence of state B (i.e., the transition path to the final state C) depend on $\Delta t$.

In the atmosphere, Rossby waves hardly propagate upward after an S-SSW (e.g., Andrews et al. 1987), which suggests that the amplitude of the effective topography $h$ should be significantly reduced after the vortex breakdown. To examine this effect, we conducted a numerical experiment in which the amplitude of $h$ was rapidly reduced after the vortex collapse. We obtained an anticyclonic state similar to $\mathrm{C}$ after the vortex breakdown (not shown), which implies that the emergence of the final state $\mathrm{C}$ is not sensitive to the amplitude of $h$ after the vortex collapse. In the atmosphere, however, a cyclonic state is reestablished after an S-SSW because of radiative cooling (e.g., Scott and Polvani 2006), which is not taken into account here. This point will be discussed in section 6 .

Liu and Scott (2015) conducted parameter sweep experiments where the topographic amplitude was increased linearly and slowly with time, as in our quasistatic experiment, by using spherical one-layer models (QG and shallow water) with a wavenumber-2 effective topography. In their experiments, two transitions similar to those in our experiment were observed. The difference from our results is that the flow field corresponding to state B oscillates more strongly (they called it the oscillating regime). This difference is likely attributable to the angular frequency of the effective topography, which is zero in our experiment.

Finally, we make a few remarks on causes for the variations of $\Gamma, E$, and $S$. The total PV $\Gamma$ and energy $E$ are varied at the two transitions $(t \sim 7000$ and 18000 days) primarily by their fluxes across $45^{\circ} \mathrm{N}$. By contrast, the two abrupt changes in the entropy $S$ are due to its fluxes and also to the viscosity effect. Particularly at the second transition, the small-scale structures develop; the entropy is transferred into smaller scales; it is finally dissipated by the numerical viscosity. These time series are not highly sensitive to the viscosity coefficient $\nu$ in (1). We obtained time series similar to those in Fig. 5a when $\nu$ was 10 times as large as the present value.

\section{Interpretations of the quasi-static experiment in terms of equilibrium statistical mechanics}

In this section, the results of the quasi-static experiment are compared with the theoretical calculations performed in section 3. In section 5a, we first confirm that the statistical mechanics theory can be applied to the polar cap over $45^{\circ}-90^{\circ} \mathrm{N}$. In section $5 \mathrm{~b}$, the PV fields in the quasi-static experiment are qualitatively compared with those of the equilibrium and quasi-stationary states. In section $5 \mathrm{c}$, we make quantitative comparisons to demonstrate that a transition occurs when the parameters (i.e., $a, \Gamma$, and $E$ ) enter the domain without an appropriate quasi-stationary state. Finally, in section 5d, a new interpretation of S-SSWs is proposed.

\section{a. Preliminaries}

The statistical mechanics theory, which predicts a steady state realized after strong PV mixing, applies only to an isolated system whose inertial time scale is much shorter than the dissipation and forcing time scales. As we stated in section $4 \mathrm{~b}$, the PV field in the quasi-static experiment is not highly sensitive to the topographic time scale $\Delta t$ (i.e., forcing time scale) or to the viscosity coefficient $\nu$, which controls the dissipation time scale. This implies that the inertial time scale (i.e., the eddy turnover time of the polar vortex) is sufficiently shorter than the dissipation and forcing time scales. In addition, the PV field is nearly steady except around the two transitions. More precisely, the components of the PV field with time scales of 100 days or less are negligible (not shown). In other words, despite the efflux of PV and energy, the polar cap north of $45^{\circ} \mathrm{N}$ can be regarded as a closed domain at a time scale of 100 days or less. Therefore, the statistical mechanics theory, namely, the variational problem (4), can be used to interpret changes in the flow-field structure over the North Pole.

\section{b. Qualitative comparisons in terms of PV fields}

In this subsection, we qualitatively compare the PV fields and suggest that the initial state $\mathrm{A}$ is interpreted as QSS 1, the intermediate state B as QSS 3, and the final state $\mathrm{C}$ as the equilibrium state. As typical snapshots, the day-4000, -11000 , and -28000 PV fields (regarded as A, B, and C, respectively) in Fig. 5b are compared with the theoretically obtained PV fields.

State $\mathrm{A}$ is cyclonic and has a large-scale structure (Fig. 5b), which suggests that A is regarded as QSS 1, because QSS 1 has the largest structure among all quasistationary states (section $3 b$ ). Figure 6a shows the PV fields obtained from the theoretical calculations using the parameters of $(a, \Gamma, E)$ from the quasi-static experiment. As expected, the PV magnitude and distribution of QSS 1 are quite similar to those of state A (Figs. 5b and 6a).

State B is cyclonic, and its PV field is slightly elongated along the lateral direction (Fig. 5b), which implies that B can be interpreted as QSS 3, because QSS 3 has the largest structure among all the quasi-stationary states having laterally long PV fields. The PV magnitude of QSS 3 is comparable to that of state B (Figs. 5b and 6a). Although the PV patch of QSS 3 is smaller than 


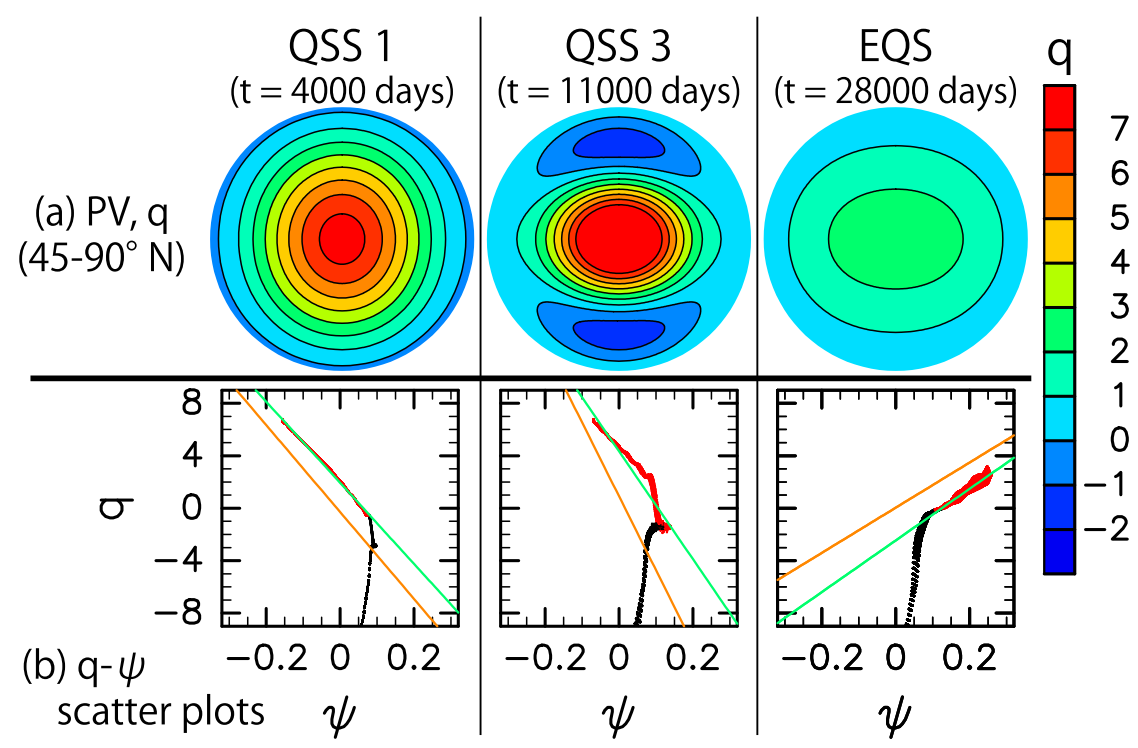

FIG. 6. (a) Theoretically calculated PV fields. Each field is obtained by giving the instantaneous parameters of $(a, \Gamma, E)$ in the quasi-static experiment. For instance, to obtain QSS 1, the parameters of $(a, \Gamma, E)$ on day 4000 are used in the theory. Each PV field is projected onto the sphere with the inverse of Lambert's map, and then it is projected again onto the plane with an orthographic projection, as for all PV fields in Fig. 5b. (b) The $q-\psi$ scatterplots in the quasi-static experiment at $t=$ (left) 4000, (middle) 11000 , and (right) 28000 days. The red dots represent the values from the grid points north of $45^{\circ} \mathrm{N}$, and the black ones represent the values from the other points. The green lines are given by the least squares fit to the red dots. Each orange line represents the $q-\psi$ relationship in (8) for the above theoretically calculated field.

that of state B, both PV fields have qualitatively similar shapes.

Only state $\mathrm{C}$ is anticyclonic (Fig. 5b), which indicates that $C$ is considered as the equilibrium state, because the equilibrium state is anticyclonic in the parameter range covered by the quasi-static experiment (section 3b). In fact, the magnitude and distribution of the equilibrium PV field are quite similar to those of state C (Figs. 5b and $6 a$ ). Even by using another theory, such as wavemean flow interaction theory, we will obtain the result that $\mathrm{PV}$ is strongly mixed because of the sufficiently high effective topography and the flow field becomes anticyclonic. However, it will then be unclear whether this state is the global equilibrium state (i.e., the entropy maximum), unless we solve the variational problem (4), because there are infinitely many steady states in the phase space (e.g., Bouchet and Venaille 2012), most of which are not entropy maxima.

There is also a good agreement on the $q-\psi$ relationship. Figure $6 \mathrm{~b}$ shows the $q-\psi$ scatterplots obtained from the quasi-static experiment. In each panel, the green line is given by the least squares fitting to the red dots, which represent the values from the grid points north of $45^{\circ} \mathrm{N}$ (the black dots show the values from the other points). There are well-defined $q-\psi$ relationships over the North Pole, and the linear fitting is valid as the first-order approximation to these relationships. The theory gives the linear $q-\psi$ relationship, namely, $q=b \psi-c$ in (8), which characterizes the equilibrium and quasi-stationary states. Figure $6 \mathrm{~b}$ also shows these theoretically calculated relationships (orange lines). The inclinations $b$ given by the theory agree well with those of the quasi-static experiment, but there is a discrepancy in the offsets $c$. This discrepancy is likely because $c$ is dependent on the latitude at the polar cap boundary. It would be possible to determine a polar cap at each time so that a theoretical value of $c$ is close to a value from the quasi-static experiment. However, such treatment is not necessary for the qualitative comparisons here.

Therefore, we expect that the state changes observed in the quasi-static experiment are understood as

$$
\begin{aligned}
\text { QSS1(state A) } & \rightarrow \text { QSS3(state B) } \\
& \rightarrow \text { Equilibrium state (state C) }
\end{aligned}
$$

The polar vortex breaks down without splitting during the transition from B to C. By contrast with the quasistatic experiment, when the topographic amplitude is increased over 1 week (section $4 \mathrm{a}$ ), state B does not 
appear, and the polar vortex collapses while splitting into two vortices during the transition from A to C.

\section{c. Quantitative comparisons in terms of transition timings}

The above discussions are qualitative and based on the instantaneous PV snapshots. In this subsection, using the time series of the total PV $\Gamma$ and energy $E$ in the quasi-static experiment, we demonstrate that the timings of the two transitions are consistent with the theoretical predictions. This result supports the above expectation of the state changes. The comparisons of transition timings are quantitative and dependent on the size of the polar cap. We properly determine the polar cap for the variational problem (4) and then discuss the transition timings.

\section{1) DETERMinAtion OF POLAR CAP BASED ON SURFZONE EDGE}

The theoretical calculations are performed within the disk on the plane, which implies that a discrepancy between the theoretical calculations and the simulation results will be larger as the polar cap becomes larger, because of the difference between the flat and spherical geometries. This suggests that the polar cap should be as small as possible.

The theory describes a flow field having the linear $q-\psi$ relationship: $q=b \psi-c$ in (8). If $q$ is nearly constant over some region, only $b=0$ is possible (i.e., $q=c=$ const) because $\psi$ is generally not constant even in such a region. Clearly, $b=0$ is inappropriate for the flow field over the North Pole, as seen in Fig. 6b. The region with nearly constant $\mathrm{PV}$ is well known as the surfzone in the stratosphere (e.g., McIntyre and Palmer 1983, 1984), and a similar region is observed here. Therefore, we define the polar cap boundary as the north edge of the surfzone.

The north edge of the surfzone is defined in two steps: (i) the latitude with the minimum $\partial \bar{q} / \partial \varphi$ is determined and denoted by $\varphi_{\min }$, where $\bar{q}$ is the zonal-mean PV, and (ii) the north (south) edge of the surfzone $\varphi_{\text {surf, }}\left(\varphi_{\text {surf,S }}\right)$ is defined as the latitude north (south) of $\varphi_{\min }$ at which $\partial \bar{q} / \partial \varphi$ first exceeds 7.0. The value of 7.0 is arbitrary, but the following results are insensitive to this value. Figure 7 a shows the time series of $\varphi_{\text {surf,N }}$ and $\varphi_{\text {surf,S }}$, which end at $t=18000$ days because of the polar vortex breakdown. Figures $7 \mathrm{~b}$ and $7 \mathrm{c}$ show the PV fields and plots of zonal-mean PV, where the vertical lines represent $\varphi_{\text {surf,N }}$ and $\varphi_{\text {surf,S }}$. Obviously, this definition of the surfzone edges captures the region with nearly constant $\mathrm{PV}$. The surfzone becomes wider as the topographic amplitude $a$ is increased. Similar results were reported by Polvani et al. (1995), who investigated the surfzone with a spherical shallow-water model including an effective topography.

We also determined the vortex boundary region by following Nash et al. (1996), where actual latitudes were used instead of equivalent latitudes because the PV field is nearly axisymmetric and monotonically increasing (Figs. $7 b, c)$. The vortex boundary region is the area between the main (polar) vortex and the surfzone, in which the vortex edge exists. We confirmed that the south edge of the vortex boundary region is quite close to the north edge of the surfzone defined above (not shown), which implies that similar results to those in Fig. 7 are obtained by the method of Nash et al. (1996).

\section{2) COMPARISONS OF TRANSITION TIMINGS}

We next discuss the transition timings. The transition from state $\mathrm{A}$ to state $\mathrm{B}$ is first investigated. This transition occurs at about $t=7000$ days (Fig. 5a), and $\varphi_{\text {surf,N }}$ is equal to about $43.6^{\circ} \mathrm{N}$ just before the transition (Figs. 7a,b). Therefore, the theoretical calculations are performed within the polar cap north of $43.6^{\circ} \mathrm{N}$. The minimum energy for the existence of any quasi-stationary state can be theoretically calculated when the topographic amplitude $a$ and the total PV $\Gamma$ are given, as shown in Fig. 3. Since we expect that the transition from A to $\mathrm{B}$ is considered as that from QSS 1 to 3, the minimum energy for QSS 1 is calculated at each time by giving the instantaneous values of $a$ and $\Gamma$ in the quasi-static experiment. Figure 8a compares the time series of the minimum energy for QSS 1 (orange) with that of the energy in the quasi-static experiment (black). The bottom panel shows the time series of the entropy $S$ in the quasistatic experiment. In Fig. 8a, the surface integrals of $\Gamma, E$, and $S$ in (5)-(7) are taken over $43.6^{\circ}-90^{\circ} \mathrm{N}$, and the offset of PV $\left[f_{\text {off }}\right.$ in (2)] is given by the Coriolis parameter at $43.6^{\circ} \mathrm{N}$. Before reaching the entropy minimum, the energy becomes lower than the minimum energy for QSS 1, which suggests that a transition occurs at about that time. In fact, associated with the decrease in the entropy, the PV field changes from a vertically to a laterally elongated shape (i.e., from state A to state B), as shown in the time series of the major-axis angle of the equivalent ellipse (Fig. 5a). These results support that state A before the first transition is regarded as QSS 1.

The entropy minimum is important here because QSS 1 is virtually a local maximum of the entropy (section 3b). The only way to increase the entropy of QSS 1 is to add a perturbation including the largest wavenumber-1 modes. In the quasi-static experiment, however, the initial PV is axisymmetric and the effective topography $h$ in (3) consists of only the wavenumber-2 component. This means that a wavenumber- 1 perturbation is never produced in the QG model, except for a 


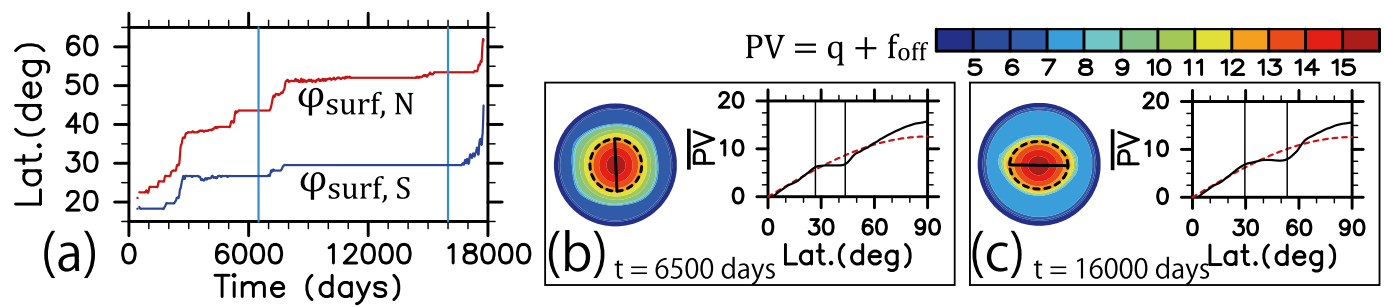

FIG. 7. (a) Time series of the north and south edges of the surfzone $\varphi_{\text {surf,N }}$ (red) and $\varphi_{\text {surf,S }}$ (blue), respectively, in the quasi-static experiment. The light-blue vertical lines represent $t=6500$ and 16000 days. The $y$-axis indicates latitude $\left({ }^{\circ} \mathrm{N}\right)$. (b), (c) PV fields over the Northern Hemisphere and plots of zonal-mean PV vs latitude at $t=(\mathrm{b}) 6500$ and (c) 16000 days in the quasi-static experiment. In the PV snapshots, the equivalent ellipses are drawn by the dashed curves and their major axes by the solid lines. In the zonal-mean PV plots, the red dashed curves represent the Coriolis parameter $f(\varphi) \equiv 2 \Omega \sin \varphi$ and the vertical lines represent $\varphi_{\text {surf,N }}$ and $\varphi_{\text {surf,S }}$. The PV offset $f_{\text {off }}$ in (2) is added to $q: \mathrm{PV} \equiv q+f_{\text {off }}$.

noise from numerical errors. Assuming that such a noise is not critical, we can regard QSS 1 as a local maximum of the entropy. In this case, the entropy will decrease in a transition from QSS 1 to another quasi-stationary state, and the entropy minimum implies that the transition is completed. We confirmed that the results here were hardly changed when a wavenumber-1 perturbation was added to the initial PV in the quasi-static experiment (not shown). Furthermore, the dynamical stability of QSS 1 (state A) was numerically investigated by directly adding a wavenumber-1 perturbation to an intermediate state regarded as QSS 1 and examining its time evolution with the spherical QG model (1) and (2). The small but finite-amplitude wavenumber-1 perturbation does not grow with time (see spp4.pdf in the supplemental material), which suggests that QSS 1 is nonlinearly stable in the sense of Lyapunov (Lyapunov stable), even though the nonlinear stability of QSS 1 against the largest wavenumber-1 Laplacian eigenmodes is not theoretically assured (section $3 b$ ). Thus, these results imply that wavenumber- 1 perturbations are not critical to the emergence and persistence of QSS 1.

The second transition from state B to state C is examined in a similar way. This transition occurs at about $t=18000$ days (Fig. 5a), and $\varphi_{\text {surf, N }}$ is equal to about $53.4^{\circ} \mathrm{N}$ just before the transition (Figs. $7 \mathrm{a}, \mathrm{c}$ ). Since we expect that the transition from $\mathrm{B}$ to $\mathrm{C}$ is considered as that from QSS 3 to the equilibrium state, the minimum energy for QSS 3 is theoretically calculated within the polar cap north of $53.4^{\circ} \mathrm{N}$ and is compared with the energy in the quasi-static experiment. Figure $8 b$ shows the result, as Fig. 8a. The predicted timing of the transition is nearly the same as that in the quasi-static experiment, which is characterized by an abrupt increase in the entropy. The result supports that state B before the second transition is interpreted as QSS 3.


FIG. 8. (a) Time series obtained from the quasi-static experiment: (top) total energy $E$ and (bottom) entropy $S$. The orange curve shows the minimum energy for QSS 1 , which is theoretically calculated by giving the instantaneous values of the topographic amplitude $a$ and total PV $\Gamma$ in the quasi-static experiment. All calculations are performed within the polar cap north of $43.6^{\circ} \mathrm{N}$. (b) As in (a), but for the minimum energy for QSS 3 , where the latitude at the polar cap boundary is $53.4^{\circ} \mathrm{N}$. 


\section{d. A new interpretation of S-SSWs in terms of equilibrium statistical mechanics}

We have demonstrated that the initial state A (cyclonic) is interpreted as QSS 1 , the intermediate state B (cyclonic) as QSS 3, and the final state C (anticyclonic) as the equilibrium state. Although the topographic time scale of 1 week is relevant to S-SSWs (Sjoberg and Birner 2012), the comparisons between the statistical mechanics theory and the quasi-static experiment have revealed that the initial state can be considered as QSS 1, which is virtually a local maximum of the entropy (section 3b). In contrast to the quasi-static experiment, when the topographic time scale is 1 week (section 4a), the polar vortex splits and breaks down during the transition from QSS 1 to the equilibrium state.

Therefore, we propose a new interpretation of S-SSWs in terms of equilibrium statistical mechanics. The S-SSW can be qualitatively understood as the transition from the cyclonic quasi-stationary state (i.e., QSS 1) toward the anticyclonic equilibrium state. The vortex splitting is observed during this transition, which is a nonequilibrium state. Without any external forcing, such as radiative cooling, the anticyclonic equilibrium state would be realized at a sufficiently long time after an S-SSW.

A typical phenomenon understood in a similar way is the transition from supercooled water to ice. Supercooled water suddenly changes into ice when some shock is given. The state of ice is the entropy maximum but that of supercooled water is a local maximum of the entropy. According to our interpretation, supercooled water corresponds to the state before an S-SSW (i.e., QSS 1). Some shock corresponds to the Rossby wave (i.e., effective topography) amplification. Ice corresponds to the state at a sufficiently long time after an S-SSW (i.e., the equilibrium state). The only difference is that QSS 1 is virtually a local maximum of the entropy, but supercooled water is exactly a local maximum. We stress that the transition described here is peculiar, as it corresponds to a transition from a local entropy maximum to a global entropy maximum. The concept of entropy reveals the analogy between the S-SSW and the transition from supercooled water to ice.

\section{Concluding remarks}

The present study has investigated vortex-split sudden stratospheric warmings (S-SSWs) from the viewpoint of equilibrium statistical mechanics. The S-SSW can be qualitatively interpreted as the transition from a cyclonic quasi-stationary state toward the anticyclonic equilibrium state. A quasi-stationary state is defined as a saddle point of the entropy, and an equilibrium state is defined as the entropy maximum. The transient state with the two split vortices is a nonequilibrium state that appears during the transition from the cyclonic quasi-stationary state toward the anticyclonic equilibrium state. This quasi-stationary state (QSS 1) is virtually a local maximum of the entropy, and only a few zonal wavenumber-1 modes can increase its entropy. Without any external forcing, such as radiative cooling, the anticyclonic equilibrium state would be realized at a sufficiently long time after an S-SSW. Our answers to questions 1 and 2 in section 1 are summarized as follows:

1) The mean state of the stratosphere accompanied by the cyclonic polar vortex is not close to an equilibrium state (anticyclonic) but is close to the dominant quasi-stationary state (i.e., QSS 1; cyclonic).

2) The S-SSW can be qualitatively interpreted as a transition from a local entropy maximum to a global maximum.

There are at least two important future themes. The first is regarding vortex-displacement SSWs (D-SSWs). It will be interesting to examine whether D-SSWs can be interpreted as a transition in terms of equilibrium statistical mechanics. Since a baroclinic structure is essential for D-SSWs (Matthewman et al. 2009; Esler and Matthewman 2011), a continuously stratified QG model is necessary for investigation. The three-dimensional version of the variational problem (4) has already been applied to geophysical fluid problems (e.g., Merryfield 1998; Venaille 2012). It will be a first step to analyze D-SSWs using this theory. Moreover, the polar vortex rapidly becomes weak at the onset of spring, which is called the stratospheric final warming (SFW), and most of these events are vortex-displacement type (Black and McDaniel 2007). There are two types of SFWs: one is more dynamically driven, and the other is more radiatively driven (Hardiman et al. 2011). The former, dynamically driven SFW, is more likely to be understood in terms of statistical mechanics because it is not applicable to a system driven mainly by radiative relaxation.

The second important theme is related to radiative cooling. Equilibrium statistical mechanics does not incorporate external forcings (e.g., radiative relaxation), which may be a serious limitation. However, statistical mechanics can be used for the qualitative analysis of forced-dissipative systems. For instance, in this study, we have shown that there are a few possible states inherent in the unforced QG system, such as the 
(anticyclonic) equilibrium state and the dominant (cyclonic) quasi-stationary state (QSS 1). The radiative cooling makes the winter stratosphere cyclonic, even though the most probable state (i.e., equilibrium state) is anticyclonic. Thus, we can make an interpretation that the radiative cooling selects QSS 1 among possible states, though its reason is unclear.

The dynamical stability of QSS 1 is important because it may be regarded as the climatological state of the winter stratosphere. We have numerically suggested that QSS 1 is nonlinearly stable (see spp4.pdf in the supplemental material,), though its stability against the largest zonal wavenumber-1 Laplacian eigenmodes is not theoretically assured. If a conserved quantity that is convex around QSS 1 in the phase space was found, the nonlinear stability of QSS 1 against any small-amplitude perturbation would be theoretically assured (e.g., Bouchet and Venaille 2012).

The radiative cooling is also vital for the reformation of the polar vortex after an S-SSW (e.g., Rong and Waugh 2004; Scott and Polvani 2006). If the transition time scale toward the equilibrium state is much longer than the radiative time scale, the cyclonic polar vortex is reestablished before the anticyclonic equilibrium state is organized. However, zonal-mean zonal winds change from westerly to easterly during an S-SSW, which means that the axisymmetric flow temporarily becomes anticyclonic. This implies that the state of the stratosphere temporarily approaches the anticyclonic equilibrium state. The QG model including a radiative relaxation may be able to be examined by using nonequilibrium statistical mechanics, which is a challenging and interesting subject to be addressed in the future to more properly describe S-SSWs.

Acknowledgments. The authors deeply appreciate the constructive comments of two anonymous reviewers. Y. Yasuda (YY) greatly appreciates the advice from Hiroshi Niino, Hisashi Nakamura, and Yohei Onuki on the presentation of the present study. YY is deeply grateful to Kaoru Sato, Masashi Kohma, Soichiro Hirano, and Arata Amemiya for discussing procedures for analyzing JRA-55. YY is also grateful to Keiichi Ishioka and Izumi Saito for giving comments on the modification of the model codes and operation of the model. We thank Editage (www. editage.jp) for English language editing. The GFD Dennou library was used to make all figures. The present study was supported by a Grant-in-Aid for Research Fellow (258466) of the JSPS and by the Leading Graduate School Program for Frontiers of Mathematical Sciences and Physics (FMSP) (Y. Yasuda). The research leading to these results has received funding from the European Research Council under the European Union's Seventh Framework Programme (FP7/2007-2013 Grant Agreement 616811) (F. Bouchet and A. Venaille).

\section{APPENDIX A}

\section{Validity of a Spherical Barotropic Quasigeostrophic Model}

We demonstrate that a spherical barotropic quasigeostrophic (QG) model [see (1) and (2)] reproduces well the evolution of the composite PV obtained from JRA-55. Similar one-layer fluid models have been used in previous studies on S-SSWs (e.g., Polvani and Waugh 2004; MirRokni et al. 2011; Matthewman and Esler 2011; Liu and Scott 2015; Scott 2016). However, to the best of our knowledge, the present study gives the first confirmation of the relevance of the barotropic QG model, through direct comparison with the reanalysis dataset. In the supplemental material (see spp1.pdf), we describe in detail the method to construct composite fields from JRA- 55 .

To compare with the QG simulations, the composite PV is constructed from JRA-55 with the following three steps: (i) The effective topography is given at each time (every $6 \mathrm{~h}$ ) by an undulation of the composite $550-\mathrm{K}$ potential temperature surface (at about $22 \mathrm{~km}$ ) and is denoted by $h_{\text {cmp }}$. The undulation is defined as the height deviation of the composite 550-K surface from its mean height $(21.7 \mathrm{~km})$, which is the zonal average at $60^{\circ} \mathrm{N}$ over the period of the composite S-SSW. (ii) The barotropic relative vorticity $\Delta \psi$ in (2) is obtained by vertically averaging the composite relative vorticity with a weight of density over 550-1300-K surfaces (about 22-39 km). (iii) The composite PV $q_{\mathrm{cmp}}$ is determined by substituting the obtained $\Delta \psi$ and $h_{\mathrm{cmp}}$ into (2).

For the numerical experiments, $h_{\mathrm{cmp}}$ is linearly interpolated in time and then set to the QG model. The initial PV is given by the composite PV $q_{\mathrm{cmp}}$ at $t=-10$ days, where the onset time is regarded as the reference of time, $t=0$ (see the supplemental material for details). Numerical integration is performed from $t=-10$ to 10 days. The following results are insensitive to the choice of the bottom potential temperature surface. Similar results were obtained when the 440-K surface (at about $17 \mathrm{~km}$ ) was used instead of the 550-K surface.

Figures A1a-c show the evolution of the effective topography $h_{\mathrm{cmp}}$, the composite PV $q_{\mathrm{cmp}}$, and the simulated PV $q$, respectively. The zonal wavenumber- 1 structure is observed in $h_{\mathrm{cmp}}$ at first, but gradually, the wavenumber-2 structure becomes dominant. The PV evolution is reproduced well by the QG model until $t=4$ days, especially 


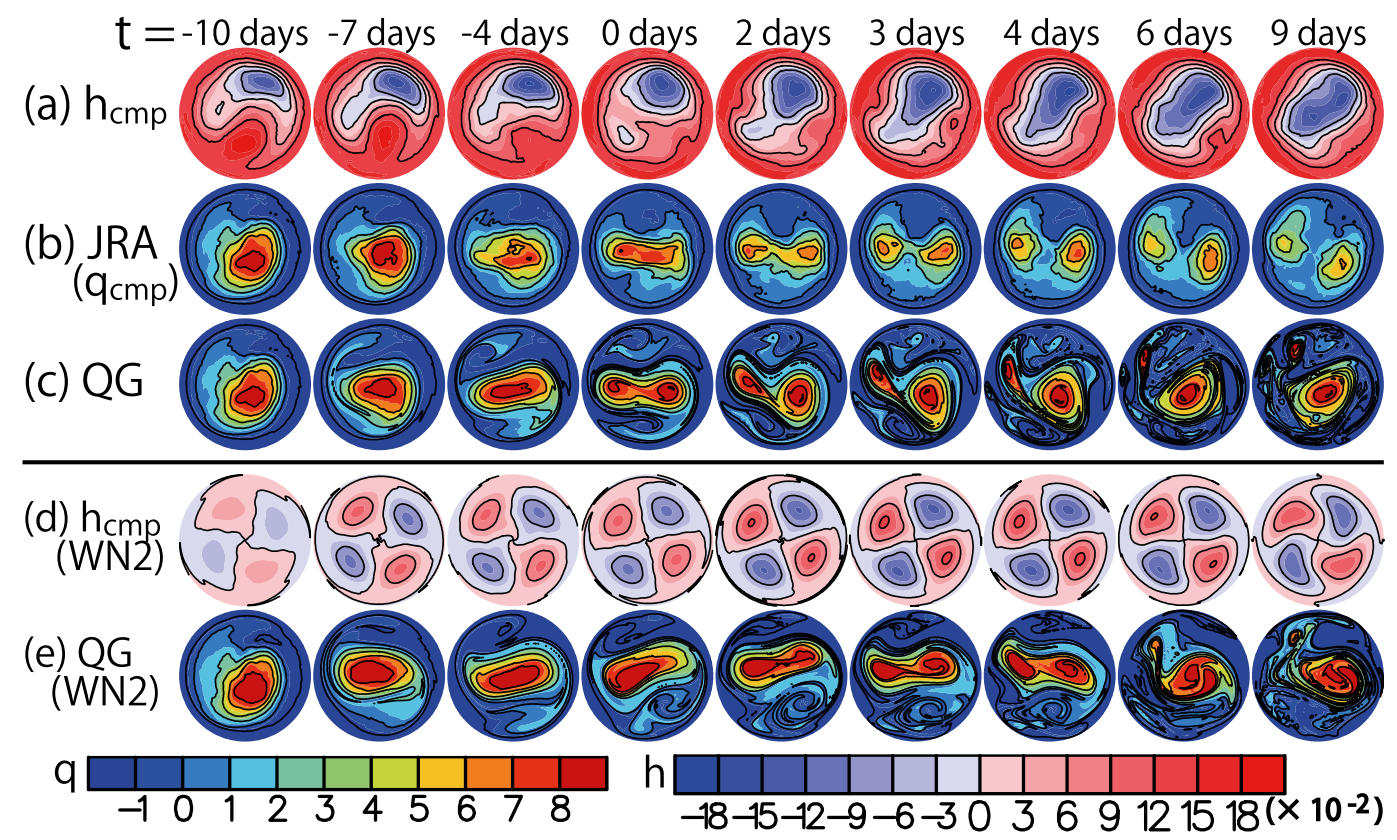

FIG. A1. Evolution over $0^{\circ}-90^{\circ} \mathrm{N}$ of (a) the effective topography $h_{\mathrm{cmp}}$ from JRA-55, (b) the composite PV $q_{\mathrm{cmp}}$ from JRA-55, (c) the simulated PV $q$ by the QG model, (d) the zonal wavenumber-2 component of $h_{\mathrm{cmp}}\left(h_{\mathrm{cmp}, 2}\right)$, and (e) the simulated PV $q$ by the QG model with only $h_{\mathrm{cmp}, 2}$ shown in (d). The magnitude of $h_{\mathrm{cmp}}$ and $h_{\mathrm{cmp}, 2}$ is nondimensionalized by $H=6.14 \mathrm{~km}$ in (a) and $0.7 \times 6.14 \mathrm{~km}$ in (d).

before and after the vortex splitting at about $t=2$ days. At a later time ( $t=6$ or 9 days), the two daughter vortices remain in the composite PV field, but only one vortex remains in the simulated field, and the other becomes quite small. This is likely due to a baroclinic structure that develops after the vortex splitting (not shown).

We further examine the zonal wavenumber component of $h_{\mathrm{cmp}}$ that is the most essential for reproducing the vortex splitting. The zonal wavenumber-0, wavenumber-1, and wavenumber- 2 components are predominant in $h_{\mathrm{cmp}}$, while the other components are negligible. Thus, only a single wavenumber (among 0,1 , or 2) component of $h_{\mathrm{cmp}}$ is given to the QG model, and the numerical integrations are conducted. In all cases, the deformation of the polar vortex is not clear, which suggests that the amplitude of each component of $h_{\mathrm{cmp}}$ is too small to reproduce a vortex splitting and that nonlinear interactions among wavenumber- $0,-1$, and -2 components produced by $h_{\text {cmp }}$ may facilitate the splitting. As the use of a smaller mean depth $H$ results in a relatively larger amplitude of $h_{\mathrm{cmp}}$ in (2), all numerical simulations are performed again with $H=\tilde{c} \times 6.14 \mathrm{~km}$, where $\tilde{c}$ is set to $0.7,0.8$, or 0.9 . The vortex split occurs only in the three cases of the wavenumber- $2 h_{\mathrm{cmp}}\left(h_{\mathrm{cmp}, 2}\right)$ with $\tilde{c}=0.7,0.8$, or 0.9 . Figures A1d and A1e show the evolution of $h_{\mathrm{cmp}, 2}$ and simulated $q$ with $\tilde{c}=0.7$, respectively. These results indicate that the zonal wavenumber- 2 component of $h_{\mathrm{cmp}}$ is the most essential feature of the effective topography that has to be retained in order to simulate the vortex splitting.

\section{APPENDIX B}

\section{Calculations Related to Variational Problem (4)}

We discuss why the negative of potential enstrophy $S$ in (7) corresponds to the mixing entropy and also give a derivation of the linear $q-\psi$ relationship (8). The discussions below basically follow the results of Naso et al. (2010). One should also see Bouchet (2008), which showed that any solution of the variational problem (4) is a solution of the more general Miller-RobertSommeria theory (the converse is not necessarily true).

\section{a. Relation between potential enstrophy and mixing entropy}

We first introduce a probability density function of PV, $\rho(\mathbf{x}, \sigma)$. The value of $\rho d \sigma d \mathbf{x}$ represents probability to observe microscopic PV whose value is between $\sigma$ and $\sigma+d \sigma$ in the infinitesimal element of $d \mathbf{x}$ around the position $\mathbf{x}$. A coarse-grained macroscopic PV field $[q]^{\text {macro }}$ is given by $[q]^{\text {macro }} \equiv \int \sigma \rho d \sigma$. Practically, a PV field observed in a numerical model should be interpreted as $[q]^{\text {macro }}$ because of the small-scale dissipation. For simplicity, we do not distinguish between $q$ and $[q]^{\text {macro }}$. 
The mixing entropy is defined as

$$
S_{\text {mix }} \equiv-\int \rho(\mathbf{x}, \sigma) \log \rho(\mathbf{x}, \sigma) d \sigma d \mathbf{x},
$$

which is proportional to the natural logarithm of the number $(\equiv N)$ of possible microscopic configurations (i.e., $S_{\text {mix }} \propto \log N$ ). Naso et al. (2010) showed that a Gaussian distribution is necessary to maximize $S_{\text {mix }}$ under the three constraints of constant total energy $E$, total PV $\Gamma$, and total microscopic potential enstrophy $\Gamma_{2}^{\text {micro: }}$

$$
\Gamma_{2}^{\text {micro }} \equiv \frac{1}{2} \int \sigma^{2} \rho(\mathbf{x}, \sigma) d \sigma d \mathbf{x} .
$$

The definitions of $E$ and $\Gamma$ are the same as (6) and (5), respectively. The Gaussian distribution is given by

$\rho(\mathbf{x}, \sigma)=\frac{1}{\sqrt{4 \pi\left(\Gamma_{2}^{\text {micro }}+S\right)}} \exp \left\{-\frac{[\sigma-q(\mathbf{x})]^{2}}{4\left(\Gamma_{2}^{\text {micro }}+S\right)}\right\}$,

where $S$ is defined by (7). The distribution $\rho$ in (B3) satisfies the constraint of $\Gamma_{2}^{\text {micro }}=(1 / 2) \int \sigma^{2} \rho d \sigma d \mathbf{x}$ (= const). Substituting (B3) into (B1), we obtain the one-to-one correspondence between $S_{\text {mix }}$ and $S$ :

$$
S_{\text {mix }}=\frac{1}{2}+\frac{1}{2} \log (4 \pi)+\frac{1}{2} \log \left(\Gamma_{2}^{\text {micro }}+S\right) .
$$

Since the natural logarithm function is strictly increasing and $\Gamma_{2}^{\text {micro }}$ is constant, $S$ has to be maximized by varying $q$ in order to maximize $S_{\text {mix }}$, which leads to the variational problem (4).

An important point for deriving (B4) is the Gaussian distribution in (B3). This result may be roughly understood as follows. When we calculate the first-order variation of some functional including $S_{\text {mix }}$, this variation will have a term proportional to $\log \rho$ originating from the variation of $S_{\text {mix }}$. In addition, when this functional includes $\Gamma_{2}^{\text {micro }}$, the obtained variation will also have a term proportional to $\sigma^{2}$. Assuming that the total variation is zero and does not include higher-order terms than $\sigma^{2}$, we will obtain a Gaussian distribution, such as (B3). The mean value of the Gaussian distribution (B3) is also important, which is equal to the (macroscopic) PV $q(\mathbf{x})$. This mean value comes from a constraint on the (macroscopic) PV. See Naso et al. (2010) for details.

\section{b. Derivation of linear $q-\psi$ relationship (8)}

According to the Lagrangian multiplier theory (e.g., Gelfand and Fomin 2000), the following first-order variation with respect to any $\delta q$ is zero at a stationary point for the variational problem (4):

$$
\delta S-b \delta E-c \delta \Gamma=0,
$$

where $\Gamma, E$, and $S$ are defined in (5), (6), and (7), respectively, and $b$ and $c$ are Lagrange multipliers. With the aid of integration by parts, $\delta E$ is changed to

$$
\begin{aligned}
\delta E=\int \delta \nabla \psi \cdot \nabla \psi d A & =-\int \psi \delta \Delta \psi d A \\
& =-\int \psi \delta q d A
\end{aligned}
$$

Therefore, (B5) is transformed to

$$
\int(-q+b \psi-c) \delta q d A=0,
$$

which yields the linear $q-\psi$ relationship (8).

\section{APPENDIX C}

\section{Details of Calculations to Obtain Equilibrium and Quasi-Stationary States}

We describe the method to obtain the equilibrium and quasi-stationary states for the variational problem (4). The discussions below basically follow the results of Venaille and Bouchet (2011b), and the same notations are used. We first introduce the complete, orthonormal basis $\left\{e_{i}\right\}_{i \in \mathbb{N}}$ of Laplacian eigenmodes on a simply connected domain such as a disk: $\Delta e_{i}=-\mu_{i} e_{i}$, where an eigenvalue $\mu_{i}$ is positive. Two subspaces are further introduced. One is composed of the Laplacian eigenmodes having zero-mean values $\left(\left\langle e_{i}^{\prime}\right\rangle=0\right)$, and the other is composed of the eigenmodes having nonzero-mean values $\left(\left\langle e_{i}^{\prime \prime}\right\rangle \neq 0\right)$, where the angle brackets denote the spatial integral over the domain, and single prime and double prime emphasize the difference between the two subspaces. In each subspace, the eigenvalues are in ascending order. The Laplacian eigenmodes and eigenvalues on a disk domain are given in appendix $\mathrm{D}$.

All quantities are expressed in terms of the coordinates $\left\{q_{i}\right\}$ [the coefficients of PV $q\left(\equiv \sum_{i} q_{i} e_{i}\right)$ ]:

$$
\begin{aligned}
\psi_{i} & =-\frac{q_{i}-h_{\mathrm{tot} i}}{\mu_{i}}, \\
\Gamma & =\sum_{i} q_{i}\left\langle e_{i}\right\rangle, \\
E & =\frac{1}{2} \sum_{i} \frac{\left(q_{i}-h_{\mathrm{tot} i}\right)^{2}}{\mu_{i}}, \text { and } \\
S & =-\frac{1}{2} \sum_{i} q_{i}^{2},
\end{aligned}
$$


where $h_{\mathrm{tot}}\left(\equiv \sum_{i} h_{\mathrm{tot} i} e_{i}\right)$ is the sum of the Coriolis parameter $\left[f-f_{0}\right.$ in (2)] and the effective topography [fh/H in (2)] and $\psi_{i}$ is a coefficient of the streamfunction $\psi\left(\equiv \sum_{i} \psi_{i} e_{i}\right)$. Note that $(\mathrm{C} 1)$ represents the PV inversion (2) given by $q \equiv \Delta \psi+f-f_{0}+f h / H \equiv \Delta \psi+h_{\text {tot }}$. When both the single prime and double prime are not attached to variables in a summation, the summation is taken over all indices of both subspaces. The expression of PV at a stationary point for the variational problem (4) is obtained by expanding the linear $q-\psi$ relationship (8) by the Laplacian eigenmodes:

$$
q_{i}=\frac{b h_{\mathrm{tot} i}-c \mu_{i}\left\langle e_{i}\right\rangle}{\mu_{i}+b} .
$$

The expressions of $\Gamma$ and $E$ at a stationary point are obtained by substituting (C5) into (C2) and (C3), respectively:

$$
\begin{aligned}
\Gamma= & -c F(b)+b \sum_{i} \frac{h_{\mathrm{tot} i}\left\langle e_{i}\right\rangle}{\mu_{i}+b} \text { and } \\
E= & {\left[\sum_{i} \frac{\mu_{i} h_{\mathrm{tot} i}^{2}}{2\left(\mu_{i}+b\right)^{2}}\right]+c\left[\sum_{i} \frac{\mu_{i} h_{\mathrm{tot} i}\left\langle e_{i}\right\rangle}{\left(\mu_{i}+b\right)^{2}}\right] } \\
& +c^{2}\left[\sum_{i} \frac{\mu_{i}\left\langle e_{i}\right\rangle^{2}}{2\left(\mu_{i}+b\right)^{2}}\right],
\end{aligned}
$$

where

$$
F(b) \equiv \sum_{i} \frac{\mu_{i}\left\langle e_{i}\right\rangle^{2}}{\mu_{i}+b} .
$$

Any stationary point given by (C5) with $b>-\mu_{1}^{\prime}$ and $-\mu^{*}$ is the solution of (4), that is, the equilibrium state, where $\mu_{1}^{\prime}$ is the smallest Laplacian eigenvalue for the zero-mean eigenmodes and $-\mu^{*}$ is the largest zero of $F(b)$ [i.e., $F\left(-\mu^{*}\right)=0$ ]. In the parameter range considered in the present study, $b$ of the equilibrium states is always larger than $-\mu_{1}^{\prime}$ and $-\mu^{*}$. See Venaille and Bouchet (2011b) for the method to calculate the equilibrium state with $b=-\mu_{1}^{\prime}$ or $-\mu^{*}$. Note that equilibrium states with $b<-\mu_{1}^{\prime}$ or $-\mu^{*}$ do not exist.

An equilibrium or a quasi-stationary state is obtained by following four steps: (i) the Lagrange multiplier $c$ is analytically obtained by solving (C6) with a given $\Gamma$; (ii) by substituting the obtained $c$ into $(\mathrm{C} 7)$, the equation $E=E(b)$ is derived; (iii) the Lagrange multiplier $b$ satisfying $E=E(b)$ is numerically calculated with a given $E$; and (iv) $q_{i}$ is computed by substituting the obtained $b$ and $c$ into (C5). When $b>-\mu_{1}^{\prime}$ and $-\mu^{*}$, the obtained $q$ is the equilibrium state. In the other cases, the obtained $q$ is a quasi-stationary state. To calculate a quasi-stationary state, an appropriate $b$ needs to be selected in the third step. For instance, to obtain QSS 1, we select $b$ at the intersection of the first branch of the energy curve $y=E(b)$ with the line of $y=E$. If such an appropriate $b$ does not exist, the corresponding quasi-stationary state does not exist. In preparing Fig. 3, we numerically checked whether an appropriate $b$ existed at each parameter point of $(a, \Gamma, E)$. In other words, a domain with existence of a quasi-stationary state is a parameter set over which the corresponding branch of the energy curve $y=E(b)$ has an intersection with $y=E$.

\section{APPENDIX D}

\section{Laplacian Eigenvalues and Eigenmodes in a Disk Domain}

We give the Laplacian eigenmodes and eigenvalues in a disk domain. A position in the domain is specified by a radius $r$ and an azimuthal angle $\theta$, where the maximum of $r$ is designated as $r_{\max }$. The Laplacian eigenmodes are given by Bessel functions of the first kind $J_{n}$ and trigonometric functions when the Dirichlet boundary condition is imposed (i.e., $e_{i}=0$ at $r=r_{\max }$ ):

$$
\left\{e_{i}^{\prime}\right\}_{i \in \mathbb{N}}=\left\{A_{n, m} J_{n}\left(\frac{\alpha_{n, m}}{r_{\max }} r\right) \sin (n \theta),\right.
$$

$$
\begin{gathered}
\left.A_{n, m} J_{n}\left(\frac{\alpha_{n, m}}{r_{\max }} r\right) \cos (n \theta)\right\}_{n, m \in \mathbb{N}}, \\
\left\{e_{i}^{\prime \prime}\right\}_{i \in \mathbb{N}}=\left\{A_{0, i} J_{0}\left(\frac{\alpha_{0, i}}{r_{\max }} r\right)\right\}_{i \in \mathbb{N}}, \text { and } \\
\left\langle e_{i}^{\prime \prime}\right\rangle=\frac{2 \sqrt{\pi} r_{\max }}{\alpha_{0, i}} \operatorname{sgn}\left[J_{1}\left(\alpha_{0, i}\right)\right],
\end{gathered}
$$

where $A_{n, m}$ is a normalization constant $\left[\left\langle\left(e_{i}\right)^{2}\right\rangle=1\right]$ and $\alpha_{n, m}$ is the $m$ th zero of $J_{n}$. The corresponding eigenvalues $\mu$ are given by $\left(\alpha_{n, m} / r_{\max }\right)^{2}$.

\section{APPENDIX E}

\section{Quadratic Form Expressing Entropy around a Stationary Point}

According to the Lagrangian multiplier theory (e.g., Gelfand and Fomin 2000), the necessary and sufficient condition that a stationary point for the variational problem (4) is a local maximum of the entropy is given by the following second-order variation, in which a perturbation $\delta q$ satisfies the two first-order constraints: 


$$
0<-\delta^{2} S+b \delta^{2} E=\frac{1}{2} \sum_{i}\left(1+\frac{b}{\mu_{i}}\right)\left(\delta q_{i}\right)^{2}
$$

$$
\text { s.t. } \delta \Gamma=0 \quad \text { and } \quad \delta E=0 .
$$

The first-order constraints are expressed in terms of $\left\{\delta q_{i}\right\}$ :

$$
\begin{aligned}
& 0=\delta \Gamma=\sum_{i}\left\langle e_{i}\right\rangle \delta q_{i}=\sum_{i}\left\langle e_{i}^{\prime \prime}\right\rangle \delta q_{i}^{\prime \prime} \quad \text { and } \\
& 0=\delta E=\sum_{i} \psi_{i} \delta q_{i},
\end{aligned}
$$

where $\delta q_{i}$ is a coefficient of $\delta q$ by Laplacian eigenmodes (see also appendix $\mathrm{C}$ ), and $\psi_{i}$ is given by substituting (C5) into (C1).

The necessary and sufficient condition (E1) is not quite easy to handle because of the two first-order constraints. We solve these two linear constraints [(E2) and (E3)] for $\delta q_{1}^{\prime \prime}$ and $\delta q_{2}^{\prime \prime}$ :

$$
\begin{aligned}
\delta q_{1}^{\prime \prime}= & \sum_{i \geq 3}\left[-\frac{\left\langle e_{i}^{\prime \prime}\right\rangle}{\left\langle e_{1}^{\prime \prime}\right\rangle}+\frac{\left\langle e_{2}^{\prime \prime}\right\rangle}{\left\langle e_{1}^{\prime \prime}\right\rangle}\left(\frac{\psi_{i}^{\prime \prime}-\psi_{1}^{\prime \prime}\left\langle e_{i}^{\prime \prime}\right\rangle\left\langle\left\langle e_{1}^{\prime \prime}\right\rangle\right.}{\psi_{2}^{\prime \prime}-\psi_{1}^{\prime \prime}\left\langle e_{2}^{\prime \prime}\right\rangle /\left\langle e_{1}^{\prime \prime}\right\rangle}\right)\right] \delta q_{i}^{\prime} \\
& +\sum_{i \geq 1} \frac{\left\langle e_{2}^{\prime \prime}\right\rangle}{\left\langle e_{1}^{\prime \prime}\right\rangle}\left(\frac{\psi_{i}^{\prime}}{\psi_{2}^{\prime \prime}-\psi_{1}^{\prime \prime}\left\langle e_{2}^{\prime \prime}\right\rangle /\left\langle e_{1}^{\prime \prime}\right\rangle}\right) \delta q_{i}^{\prime} \equiv \sum_{i} B_{i} \delta q_{i}
\end{aligned}
$$

and

$$
\begin{aligned}
\delta q_{2}^{\prime \prime}= & -\sum_{i \geq 3}\left(\frac{\psi_{i}^{\prime \prime}-\psi_{1}^{\prime \prime}\left\langle e_{i}^{\prime \prime}\right\rangle /\left\langle e_{1}^{\prime \prime}\right\rangle}{\psi_{2}^{\prime \prime}-\psi_{1}^{\prime \prime}\left\langle e_{2}^{\prime \prime}\right\rangle /\left\langle e_{1}^{\prime \prime}\right\rangle}\right) \delta q_{i}^{\prime \prime} \\
& -\sum_{i \geq 1}\left(\frac{\psi_{i}^{\prime}}{\psi_{2}^{\prime \prime}-\psi_{1}^{\prime \prime}\left\langle e_{2}^{\prime \prime}\right\rangle /\left\langle e_{1}^{\prime \prime}\right\rangle}\right) \delta q_{i}^{\prime} \equiv \sum_{i} C_{i} \delta q_{i} .
\end{aligned}
$$

Substituting $\delta q_{1}^{\prime \prime}$ and $\delta q_{2}^{\prime \prime}$ into (E1), we obtain the quadratic form expressing the second-order variation $[(\mathrm{E} 1)]$, in which the two first-order constraints are incorporated:

$$
2\left(-\delta^{2} S+b \delta^{2} E\right)=\sum_{\text {other } i^{\prime}} \delta q_{i}^{\prime 2}\left(1+\frac{b}{\mu_{i}^{\prime}}\right)+\sum_{i, j} \underbrace{\left[\delta_{i j}\left(1+\frac{b}{\mu_{i}}\right)+B_{i} B_{j}\left(1+\frac{b}{\mu_{1}^{\prime \prime}}\right)+C_{i} C_{j}\left(1+\frac{b}{\mu_{2}^{\prime \prime}}\right)\right]}_{Q_{i j}} \times \delta q_{i} \delta q_{j},
$$

where $\delta_{i j}$ is a Kronecker delta and other $i^{\prime}$ means all indices of the zero-mean eigenmodes whose $\psi_{i}^{\prime}$ are zero (i.e., whose $B_{i}$ and $C_{i}$ are zero). The quadratic form (E6) is decomposed into the first sum consisting of the diagonal matrix and the second sum consisting of the symmetric matrix $\mathbf{Q}$. In the present study, the second sum is given by the components having wavenumber 0 or 2 , and the other components contribute to the first sum. The quadratic form (E6) expresses the entropy surface around a stationary point in the phase space. When (E6) is positive definite, the condition (E1) is satisfied, and the stationary point is a local maximum of the entropy (i.e., dynamically and nonlinearly stable).

The definiteness of the quadratic form (E6) is examined in the following three steps: (i) the definiteness of $\mathbf{Q}$ is checked by numerically computing the eigenvalues of $\mathbf{Q}$; (ii) the value of $b$ is compared with the eigenvalues $\mu_{i}^{\prime}$, and the first sum in (E6) is positive for any $\delta q$, if and only if $-\mu_{1}^{\prime}<b$, where $\mu_{1}$ is the Laplacian eigenvalue of the largest wavenumber- 1 modes [ $n=1$ and $m=1$ in (D1)]; and (iii) if $\mathbf{Q}$ is positive definite and if $-\mu_{1}^{\prime}<b$, the quadratic form (E6) is positive definite, and the stationary point is a local maximum of the entropy. Clearly, any stationary point with $b<-\mu_{1}^{\prime}$ does not satisfy this condition; therefore, it is a saddle point of the entropy. Naso et al. (2010) showed the same result through a different method. They found a specific first-order perturbation $\delta q$ satisfying the first-order constraints. Substituting it into $-\delta^{2} S+b \delta^{2} E$, they showed that the condition (E1) is not satisfied for a stationary point with $b<-\mu_{1}^{\prime}$.

The uniqueness of QSS 1 comes from the positive definiteness of the symmetric matrix $\mathbf{Q}$. This means that QSS 1 is a local maximum of the entropy if $-\mu_{1}^{\prime}<b$. In other words, the structure of the entropy surface around QSS 1 is determined only by the inequality of $b$ and $-\mu_{1}^{\prime}$. Furthermore, the value of $\mu_{1}^{\prime}$ is determined only by the shape of a domain.

In a disk domain, $-\mu^{*}<-\mu_{1}^{\prime}$ holds. The range of $b$ for QSS 1 is analytically obtained as $-\mu_{2}^{\prime}<b<-\mu^{*}$, where $\mu_{2}^{\prime}$ is the Laplacian eigenvalue of the largest wavenumber-2 modes [ $n=2$ and $m=1$ in (D1)]. Therefore, any $b$ of QSS 1 is smaller than $-\mu_{1}^{\prime}$, and QSS 1 is always a saddle point of the entropy. In the quadratic form (E6), the largest wavenumber-1 modes of a perturbation, $\delta q_{1}^{\prime}$, are included only in the first sum. If a perturbation $\delta q$ does not have these components, the quadratic form (E6) is positive. By contrast, in a rectangular domain, QSS 1 satisfies $-\mu_{1}^{\prime}<b$, depending on the parameters of $a, \Gamma$, and $E$. Therefore, QSS 1 can be a local maximum of the entropy (i.e., metastable; not shown). 


\section{REFERENCES}

Andrews, D. G., J. R. Holton, and C. B. Leovy, 1987: Middle Atmosphere Dynamics. International Geophysics Series, Vol. 40, Academic Press, 489 pp.

Baldwin, M. P., and T. J. Dunkerton, 2001: Stratospheric harbingers of anomalous weather regimes. Science, 294, 581-584, doi:10.1126/science.1063315.

Birner, T., and P. D. Williams, 2008: Sudden stratospheric warmings as noise-induced transitions. J. Atmos. Sci., 65, 3337-3343, doi:10.1175/2008JAS2770.1.

Black, R. X., and B. A. McDaniel, 2007: The dynamics of Northern Hemisphere stratospheric final warming events. J. Atmos. Sci., 64, 2932-2946, doi:10.1175/JAS3981.1.

Bouchet, F., 2008: Simpler variational problems for statistical equilibria of the 2D Euler equation and other systems with long range interactions. Physica D, 237, 1976-1981, doi:10.1016/j.physd.2008.02.029.

— ter's great red spot as maximum-entropy structures. J. Fluid Mech., 464, 165-207, doi:10.1017/S0022112002008789.

- , and A. Venaille, 2012: Statistical mechanics of twodimensional and geophysical flows. Phys. Rep., 515, 227-295, doi:10.1016/j.physrep.2012.02.001.

Butler, A. H., D. J. Seidel, S. C. Hardiman, N. Butchart, T. Birner, and A. Match, 2015: Defining sudden stratospheric warmings. Bull. Amer. Meteor. Soc., 96, 1913-1928, doi:10.1175/ BAMS-D-13-00173.1.

Chao, W. C., 1985: Sudden stratospheric warmings as catastrophes. J. Atmos. Sci., 42, 1631-1646, doi:10.1175/ 1520-0469(1985)042<1631:SSWAC >2.0.CO;2.

Charlton, A. J., and L. M. Polvani, 2007: A new look at stratospheric sudden warmings. Part I: Climatology and modeling benchmarks. J. Climate, 20, 449-469, doi:10.1175/JCLI3996.1.

Chavanis, P. H., and J. Sommeria, 1996: Classification of selforganized vortices in two-dimensional turbulence: The case of a bounded domain. J. Fluid Mech., 314, 267-297, doi:10.1017/S0022112096000316.

Christiansen, B., 2000: Chaos, quasiperiodicity, and interannual variability: Studies of a stratospheric vacillation model. J. Atmos. Sci., 57, 3161-3173, doi:10.1175/1520-0469(2000) $057<3161$ :CQAIVS $>2.0 . \mathrm{CO} ; 2$.

Ellis, R. S., K. Haven, and B. Turkington, 2002: Nonequivalent statistical equilibrium ensembles and refined stability theorems for most probable flows. Nonlinearity, 15, 239-255, doi:10.1088/0951-7715/15/2/302.

Esler, J. G., 2008: The turbulent equilibration of an unstable baroclinic jet. J. Fluid Mech., 599, 241-268, doi:10.1017/ S0022112008000153.

— , and R. K. Scott, 2005: Excitation of transient Rossby waves on the stratospheric polar vortex and the barotropic sudden warming. J. Atmos. Sci., 62, 3661-3682, doi:10.1175/JAS3557.1.

_ , and N. J. Matthewman, 2011: Stratospheric sudden warmings as self-tuning resonances. Part II: Vortex displacement events. J. Atmos. Sci., 68, 2505-2523, doi:10.1175/JAS-D-11-08.1.

Gelfand, I. M., and S. V. Fomin, 2000: Calculus of Variations. Dover Publications, $240 \mathrm{pp}$.

Harada, Y., and Coauthors, 2016: The JRA-55 Reanalysis: Representation of atmospheric circulation and climate variability. J. Meteor. Soc. Japan, 94, 269-302, doi:10.2151/jmsj.2016-015.

Hardiman, S. C., and Coauthors, 2011: Improved predictability of the troposphere using stratospheric final warmings. J. Geophys. Res., 116, D18113, doi:10.1029/2011JD015914.
Haynes, P., 2005: Stratospheric dynamics. Annu. Rev. Fluid Mech., 37, 263-293, doi:10.1146/annurev.fluid.37.061903.175710.

Hitchcock, P., and I. R. Simpson, 2014: The downward influence of stratospheric sudden warmings. J. Atmos. Sci., 71, 3856-3876, doi:10.1175/JAS-D-14-0012.1.

Holton, J. R., and C. Mass, 1976: Stratospheric vacillation cycles. J. Atmos. Sci., 33, 2218-2225, doi:10.1175/ 1520-0469(1976)033<2218:SVC >2.0.CO;2.

Ishioka, K., 2013: ISPACK Version 1.0.2. Geophysical Fluid Dynamics Dennou Club, http://www.gfd-dennou.org/arch/ispack/ index.htm.en.

Japan Meteorological Agency, 2014: JRA-55-The Japanese 55-year Reanalysis. Japan Meteorological Agency, accessed 11 June 2014, http://jra.kishou.go.jp/JRA-55/index_en.html.

Kidston, J., A. A. Scaife, S. C. Hardiman, D. M. Mitchell, N. Butchart, M. P. Baldwin, and L. J. Gray, 2015: Stratospheric influence on tropospheric jet streams, storm tracks and surface weather. Nat. Geosci., 8, 433-440, doi:10.1038/ngeo2424.

Kobayashi, S., and Coauthors, 2015: The JRA-55 Reanalysis: General specifications and basic characteristics. J. Meteor. Soc. Japan, 93, 5-48, doi:10.2151/jmsj.2015-001.

Limpasuvan, V., D. W. J. Thompson, and D. L. Hartmann, 2004: The life cycle of the Northern Hemisphere sudden stratospheric warmings. J. Climate, 17, 2584-2596, doi:10.1175/ 1520-0442(2004)017<2584:TLCOTN>2.0.CO;2.

Liu, Y. S., and R. K. Scott, 2015: The onset of the barotropic sudden warming in a global model. Quart. J. Roy. Meteor. Soc., 141, 2944-2955, doi:10.1002/qj.2580.

Majda, A., and X. Wang, 2006: Nonlinear Dynamics and Statistical Theories for Basic Geophysical Flows. Cambridge University Press, $564 \mathrm{pp}$.

Matsuno, T., 1971: A dynamical model of the stratospheric sudden warming. J. Atmos. Sci., 28, 1479-1494, doi:10.1175/ 1520-0469(1971)028<1479:ADMOTS>2.0.CO;2.

Matthewman, N. J., and J. G. Esler, 2011: Stratospheric sudden warmings as self-tuning resonances. Part I: Vortex splitting events. J. Atmos. Sci., 68, 2481-2504, doi:10.1175/JAS-D-11-07.1.

, A. J. Charlton, and L. M. Polvani, 2009: A new look at stratospheric sudden warmings. Part III: Polar vortex evolution and vertical structure. J. Climate, 22, 1566-1585, doi:10.1175/ 2008JCLI2365.1.

Maycock, A. C., and P. Hitchcock, 2015: Do split and displacement sudden stratospheric warmings have different annular mode signatures? Geophys. Res. Lett., 42, $10943-10$ 951, doi:10.1002/ 2015 GL066754.

McIntyre, M. E., and T. N. Palmer, 1983: Breaking planetary waves in the stratosphere. Nature, 305, 593-600, doi:10.1038/305593a0. , and - 1984: The "surf zone" in the stratosphere. J. Atmos. Terr. Phys., 46, 825-849, doi:10.1016/0021-9169(84)90063-1.

Merryfield, W. J., 1998: Effects of stratification on quasigeostrophic inviscid equilibria. J. Fluid Mech., 354, 345-356, doi:10.1017/S0022112097007684.

Miller, J., 1990: Statistical mechanics of Euler equations in two dimensions. Phys. Rev. Lett., 65, 2137-2140, doi:10.1103/ PhysRevLett.65.2137.

MirRokni, S. M., A. R. Mohebalhojeh, and D. G. Dritschel, 2011: Revisiting vacillations in shallow-water models of the stratosphere using potential-vorticity-based numerical algorithms. J. Atmos. Sci., 68, 1007-1022, doi:10.1175/2011JAS3622.1.

Mitchell, D. M., L. J. Gray, J. Anstey, M. P. Baldwin, and A. J. Charlton, 2013: The influence of stratospheric vortex displacements and splits on surface climate. J. Climate, 26, 2668-2682, doi:10.1175/JCLI-D-12-00030.1. 
Monahan, A. H., J. C. Fyfe, and L. Pandolfo, 2003: The vertical structure of wintertime climate regimes of the Northern Hemisphere extratropical atmosphere. J. Climate, 16, 2005-2021, doi:10.1175/1520-0442(2003)016<2005:TVSOWC >2.0.CO;2.

Nakagawa, K. I., and K. Yamazaki, 2006: What kind of stratospheric sudden warming propagates to the troposphere? Geophys. Res. Lett., 33, L04801, doi:10.1029/2006GL025719.

Nash, E. R., P. A. Newman, J. E. Rosenfield, and M. R. Schoeberl, 1996: An objective determination of the polar vortex using Ertel's potential vorticity. J. Geophys. Res., 101, 9471-9478, doi:10.1029/96JD00066.

Naso, A., P. H. Chavanis, and B. Dubrulle, 2010: Statistical mechanics of two-dimensional Euler flows and minimum enstrophy states. Eur. Phys. J., 77B, 187-212, doi:10.1140/epjb/e2010-00269-0.

Plumb, R. A., 1981a: Forced waves in a baroclinic shear flow. Part 2: Damped and undamped response to weak nearresonant forcing. J. Atmos. Sci., 38, 1856-1869, doi:10.1175/ 1520-0469(1981)038<1856:FWIABS > 2.0.CO;2.

,1981b: Instability of the distorted polar night vortex: A theory of stratospheric warmings. J. Atmos. Sci., 38, 2514-2531, doi:10.1175/1520-0469(1981)038<2514:IOTDPN>2.0.CO;2.

Polvani, L. M., and D. W. Waugh, 2004: Upward wave activity flux as a precursor to extreme stratospheric events and subsequent anomalous surface weather regimes. J. Climate, 17, 3548-3554, doi:10.1175/1520-0442(2004)017<3548:UWAFAA >2.0.CO;2.

,-- , and R. A. Plumb, 1995: On the subtropical edge of the stratospheric surf zone. J. Atmos. Sci., 52, 1288-1309, doi:10.1175/1520-0469(1995)052<1288:OTSEOT>2.0.CO;2.

Prieto, R., and W. H. Schubert, 2001: Analytical predictions for zonally symmetric equilibrium states of the stratospheric polar vortex. J. Atmos. Sci., 58, 2709-2728, doi:10.1175/ 1520-0469(2001)058<2709:APFZSE > 2.0.CO;2.

_ J. P. Kossin, and W. H. Schubert, 2001: Symmetrization of lopsided vorticity monopoles and offset hurricane eyes. Quart. J. Roy. Meteor. Soc., 127, 2307-2327, doi:10.1002/qj.49712757706.

Robert, R., 1991: A maximum-entropy principle for twodimensional perfect fluid dynamics. J. Stat. Phys., 65, 531-553, doi:10.1007/BF01053743.

— dimensional flows. J. Fluid Mech., 229, 291-310, doi:10.1017/ S0022112091003038.

Rong, P.-P., and D. W. Waugh, 2004: Vacillations in a shallowwater model of the stratosphere. J. Atmos. Sci., 61, 1174-1185, doi:10.1175/1520-0469(2004)061<1174:VIASMO>2.0.CO;2.

Ruzmaikin, A., J. Lawrence, and C. Cadavid, 2003: A simple model of stratospheric dynamics including solar variability. J. Climate, 16, 1593-1600, doi:10.1175/1520-0442-16.10.1593.
Salmon, R., 1998: Lectures on Geophysical Fluid Dynamics. Oxford University Press, $378 \mathrm{pp}$.

Scott, R. K., 2016: A new class of vacillations of the stratospheric polar vortex. Quart. J. Roy. Meteor. Soc., 142, 1948-1957, doi:10.1002/qj.2788.

— stratosphere. Part I: Time-independent forcing. J. Atmos. Sci., 63, 2758-2776, doi:10.1175/JAS3797.1.

Seviour, W. J. M., D. M. Mitchell, and L. J. Gray, 2013: A practical method to identify displaced and split stratospheric polar vortex events. Geophys. Res. Lett., 40, 5268-5273, doi:10.1002/grl.50927.

Sjoberg, J. P., and T. Birner, 2012: Transient tropospheric forcing of sudden stratospheric warmings. J. Atmos. Sci., 69, 3420-3432, doi:10.1175/JAS-D-11-0195.1.

_ , and _ 2014: Stratospheric wave-mean flow feedbacks and sudden stratospheric warmings in a simple model forced by upward wave activity flux. J. Atmos. Sci., 71, 4055-4071, doi:10.1175/JAS-D-14-0113.1.

Tung, K. K., and R. S. Lindzen, 1979a: A theory of stationary long waves. Part I: A simple theory of blocking. Mon. Wea. Rev., 107, 714-734, doi:10.1175/1520-0493(1979)107<0714:ATOSLW>2.0.CO;2.

_ and _ 1979b: A theory of stationary long waves. Part II: Resonant Rossby waves in the presence of realistic vertical shears. Mon. Wea. Rev., 107, 735-750, doi:10.1175/1520-0493 (1979) $107<0735$ :ATOSLW>2.0.CO;2.

Vallis, G. K., 2006: Atmospheric and Oceanic Fluid Dynamics: Fundamentals and Large-Scale Circulation. Cambridge University Press, $745 \mathrm{pp}$.

Venaille, A., 2012: Bottom-trapped currents as statistical equilibrium states above topographic anomalies. J. Fluid Mech., 699, 500-510, doi:10.1017/jfm.2012.146.

_ bicritical points for two-dimensional flows and geophysical flows. Phys. Rev. Lett., 102, 104501, doi:10.1103/PhysRevLett.102.104501.

— , and —, 2011a: Ocean rings and jets as statistical equilibrium states. J. Phys. Oceanogr., 41, 1860-1873, doi:10.1175/ 2011JPO4583.1.

_ , and — 2011b: Solvable phase diagrams and ensemble inequivalence for two-dimensional and geophysical turbulent flows. J. Stat. Phys., 143, 346-380, doi:10.1007/s10955-011-0168-0.

Waugh, D. W., and L. M. Polvani, 2010: Stratospheric polar vortices. The Stratosphere: Dynamics, Transport, and Chemistry, Geophys. Monogr., Vol. 190, Amer. Geophys. Union, 43-57, doi:10.1002/9781118666630.ch3.

Yoden, S., 1987: Bifurcation properties of a stratospheric vacillation model. J. Atmos. Sci., 44, 1723-1733, doi:10.1175/ 1520-0469(1987)044<1723:BPOASV>2.0.CO;2. 